\title{
Radical Benzylation of Quinones via C-H Abstraction
}

Jordan D. Galloway, Duy N. Mai, and Ryan D. Baxter*

Department of Chemistry and Chemical Biology, University of California, Merced 5200 North Lake Road Merced, CA 95348

\section{Supporting Information}

Experimental procedures for ${ }^{19} \mathrm{~F}$ NMR studies and copies of NMR spectra for isolated compounds.

\section{Table of Contents}

Representative Experimental Procedure for ${ }^{19} \mathrm{~F}-\mathrm{NMR}$ Data $\quad$ S2

Functionalization reactions $\quad$ S4

Solvent Effect on Reaction $\quad$ S4

Attempts towards other $\mathrm{C}-\mathrm{H}$ radical precursors and heterocycles $\quad$ S5

$\begin{array}{ll}\text { Spectroscopic Data for Reported Compounds } & \text { S7 }\end{array}$ 


\section{${ }^{19}$ F-NMR studies}

Representative Experimental Procedure for ${ }^{19} \mathrm{~F}-\mathrm{NMR}$ Data

The threads of a $4 \mathrm{~mL}$ borosilicate scintillation vial were thoroughly taped with Teflon tape. To this vial containing a stir bar was added Selectfluor ${ }^{\circledR}(354 \mathrm{mg}, 1.0 \mathrm{mmol})$ and $\mathrm{AgNO}_{3}(17.0 \mathrm{mg}, .10 \mathrm{mmol})$. Dichloroethane $(2 \mathrm{~mL})$ and $\mathrm{H}_{2} \mathrm{O}(2 \mathrm{~mL})$ were then added to the vial. The solution was allowed to stir at $50^{\circ} \mathrm{C}$ for $24 \mathrm{~h}$. Using a glass microsyringe, $200 \mu \mathrm{L}$ aliquots were taken from the aqueous phase at specified time points and diluted with $400 \mu \mathrm{L}$ of $\mathrm{D}_{2} \mathrm{O}$. Decrease in Selectfluor concentration were determined by ${ }^{19} \mathrm{~F}-\mathrm{NMR}$. All ${ }^{19} \mathrm{~F}$-NMR experiments were compared to $\alpha, \alpha, \alpha$-Trifluorotoluene as standard which was added to the NMR tube in a sealed capillary tube. All data represented in Figure $4 \mathrm{~A}-\mathrm{C}$ were acquired in an analogous fashion. 







\section{Functionalization reactions}

Solvent Effect on Reaction<smiles>O=C1C=CC(=O)C=C1</smiles><smiles>Cc1ccc(C)cc1</smiles>

2 equiv. Selectfluor $20 \mathrm{~mol} \% \mathrm{Ag}(4-\mathrm{OMePy}){ }_{2} \mathrm{NO}_{3}$

Solvent $(1: 1), 50^{\circ} \mathrm{C}$ $24 \mathrm{~h}$

1 equiv. 10 equiv.

$$
24 \mathrm{~h}
$$


\begin{tabular}{cc}
\hline Entry & Deviation \\
1 & \\
1 & \\
2 & \\
3 & \\
4 & \\
5 &
\end{tabular}

from standard
DCE
DCE / Water
Acetone
Acetone / Wat
MeCN
MeCN / Wate

\section{conditions}

1

A1

Yield of $1^{\mathrm{a}} \quad$ Yield of A1

no reaction no reaction

$73 \%(71 \%)$

$2 \%$

$14 \%$

$7 \%$

$42 \% \quad 7 \%$

$19 \% \quad 20 \%$

$19 \% \quad 5 \%$

a ${ }^{1} \mathrm{H}-\mathrm{NMR}$ yields to 1,3,5-trimethoxybenzene, values in parentheses indicate isolated yield. 
Attempts towards other $\mathrm{C}-\mathrm{H}$ radical precursors and heterocycles
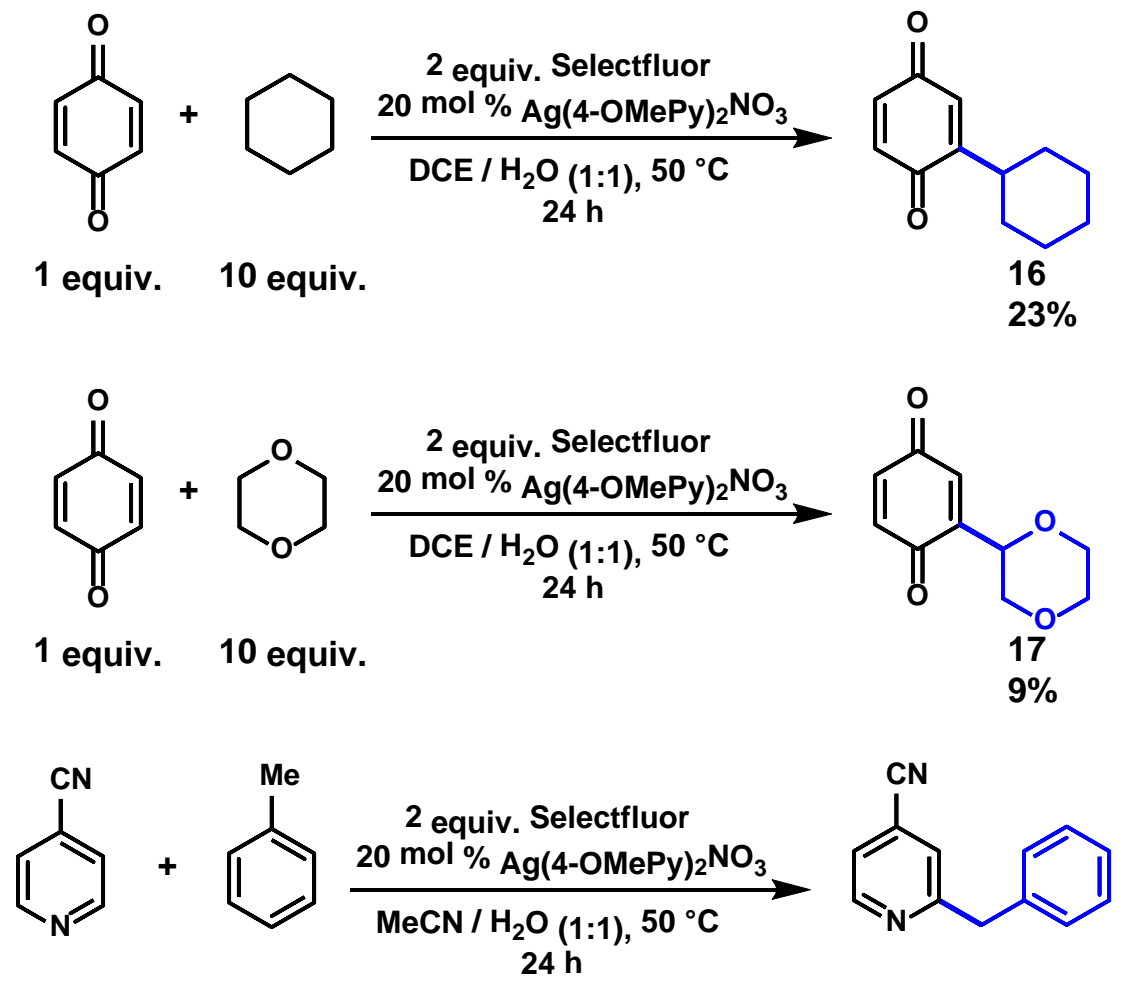

1 equiv. $\quad 10$ equiv.

18

$17 \%$



1 equiv. $\quad 10$ equiv.

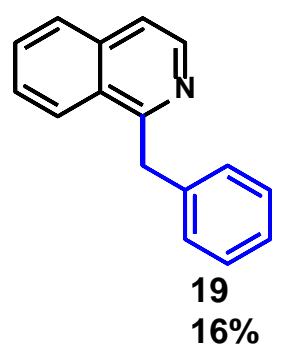


[1,1'-bi(cyclohexane)]-3,6-diene-2,5-dione (16). The general procedure was employed using 1,4-benzoquinone (22 mg, $0.2 \mathrm{mmol}$ ) and cyclohexane (216 $\mu \mathrm{L}, 2.0 \mathrm{mmol})$. The reaction afforded $\mathbf{1 6}$ (8.7 mg, 23\% yield) as a yellow oil separated by silica gel (5\% ethyl acetate in hexanes). The data matches those previously reported. ${ }^{11} \mathbf{H}$ NMR $\left(500 \mathrm{MHz}, \mathrm{CDCl}_{3}\right): 6.75$ (d, $\left.J=10.1 \mathrm{~Hz}, 1 \mathrm{H}\right), 6.69$ (dd, $\left.J=10.1,2.5 \mathrm{~Hz}, 1 \mathrm{H}\right), 6.50$ (dd, $\left.J=2.5,1.1 \mathrm{~Hz}, 1 \mathrm{H}\right), 2.75-2.64(\mathrm{~m}, 1 \mathrm{H}), 1.85-1.72(\mathrm{~m}, 5 \mathrm{H}), 1.40(\mathrm{qt}, J=12.7$, $3.2 \mathrm{~Hz}, 2 \mathrm{H}), 1.27-1.11(\mathrm{~m}, 3 \mathrm{H})$.

2-(1,4-dioxan-2-yl)cyclohexa-2,5-diene-1,4-dione (17). The general procedure was employed using 1,4-benzoquinone (22 mg, $0.2 \mathrm{mmol})$ and 1,4-dioxane (170 $\mu \mathrm{L}$, 2.0 mmol). The reaction afforded $\mathbf{1 7}$ (3.5 mg, $9 \%$ yield) as a yellow oil separated by silica gel (5\% ethyl acetate in hexanes). ${ }^{1} \mathbf{H} \mathbf{~ N M R}(500 \mathrm{MHz}, \mathrm{CDCl} 3)$ : 6.89 (dd, $J=2.7$, $1.4 \mathrm{~Hz}, 1 \mathrm{H}$ ), $6.77-6.71$ (m, $J=1.2 \mathrm{~Hz}, 2 \mathrm{H}), 4.67$ (ddd, $J=9.6,2.6,1.8 \mathrm{~Hz}, 1 \mathrm{H}), 4.05$ (dd, $J=11.1,2.7 \mathrm{~Hz}, 1 \mathrm{H}$ ), 3.90 (dtd, $J=14.5,11.8,3.0 \mathrm{~Hz}, 2 \mathrm{H}), 3.80$ (dd, $J=11.7$, $2.6 \mathrm{~Hz}, 1 \mathrm{H}$ ), 3.64 (td, $J=11.5,3.3 \mathrm{~Hz}, 1 \mathrm{H}$ ), 3.20 (dd, $J=11.1,9.6 \mathrm{~Hz}, 1 \mathrm{H}$ ).

2-benzylisonicotinonitrile (18). The general procedure was modified using MeCN instead of DCE as the organic solvent using 4-cyanopyridine (21 mg, $0.2 \mathrm{mmol}$ ) and toluene (213 $\mu \mathrm{L}, 2.0 \mathrm{mmol})$. The reaction afforded 18 ( $6.6 \mathrm{mg}, 17 \%$ yield) as a yellow oil separated by silica gel (30\% ethyl acetate in hexanes). The data matches those previously reported. ${ }^{2}{ }^{1} \mathbf{H}$ NMR (500 MHz, $\left.\mathrm{CDCl}_{3}\right): 8.73$ (d, $\left.J=5.0 \mathrm{~Hz}, 1 \mathrm{H}\right), 7.39-7.23$ (m, 8H), $4.22(\mathrm{~s}, 2 \mathrm{H})$.

1-benzylisoquinoline (19). The general procedure was using MeCN instead of DCE as the organic solvent using isoquinoline (24 $\mu \mathrm{L}, 0.2 \mathrm{mmol})$ and toluene (213 $\mu \mathrm{L}$, 2.0 mmol). The reaction afforded 19 ( $7.0 \mathrm{mg}, 16 \%$ yield) as a yellow oil separated by silica gel (30\% ethyl acetate in hexanes). The data matches those previously reported. ${ }^{\mathrm{Xx}}$ ${ }^{1} \mathbf{H}$ NMR $\left(500 \mathrm{MHz}, \mathrm{CDCl}_{3}\right): 8.43(\mathrm{~d}, J=5.7 \mathrm{~Hz}, 1 \mathrm{H}), 8.10(\mathrm{~d}, J=8.5 \mathrm{~Hz}, 1 \mathrm{H}), 7.76(\mathrm{~d}, J=8.2 \mathrm{~Hz}, 1 \mathrm{H}), 7.59(\mathrm{t}, J=7.5 \mathrm{~Hz}, 1 \mathrm{H}), 7.53(\mathrm{~d}, J=5.8 \mathrm{~Hz}, 1 \mathrm{H}), 7.48(\mathrm{t}, J=7.5$ $\mathrm{Hz}, 1 \mathrm{H}), 7.19$ (dt, $J=11.1,7.6 \mathrm{~Hz}, 4 \mathrm{H}), 7.10$ (t, $J=7.1 \mathrm{~Hz}, 1 \mathrm{H}), 4.64$ (s, 2H). 
Spectral Data 

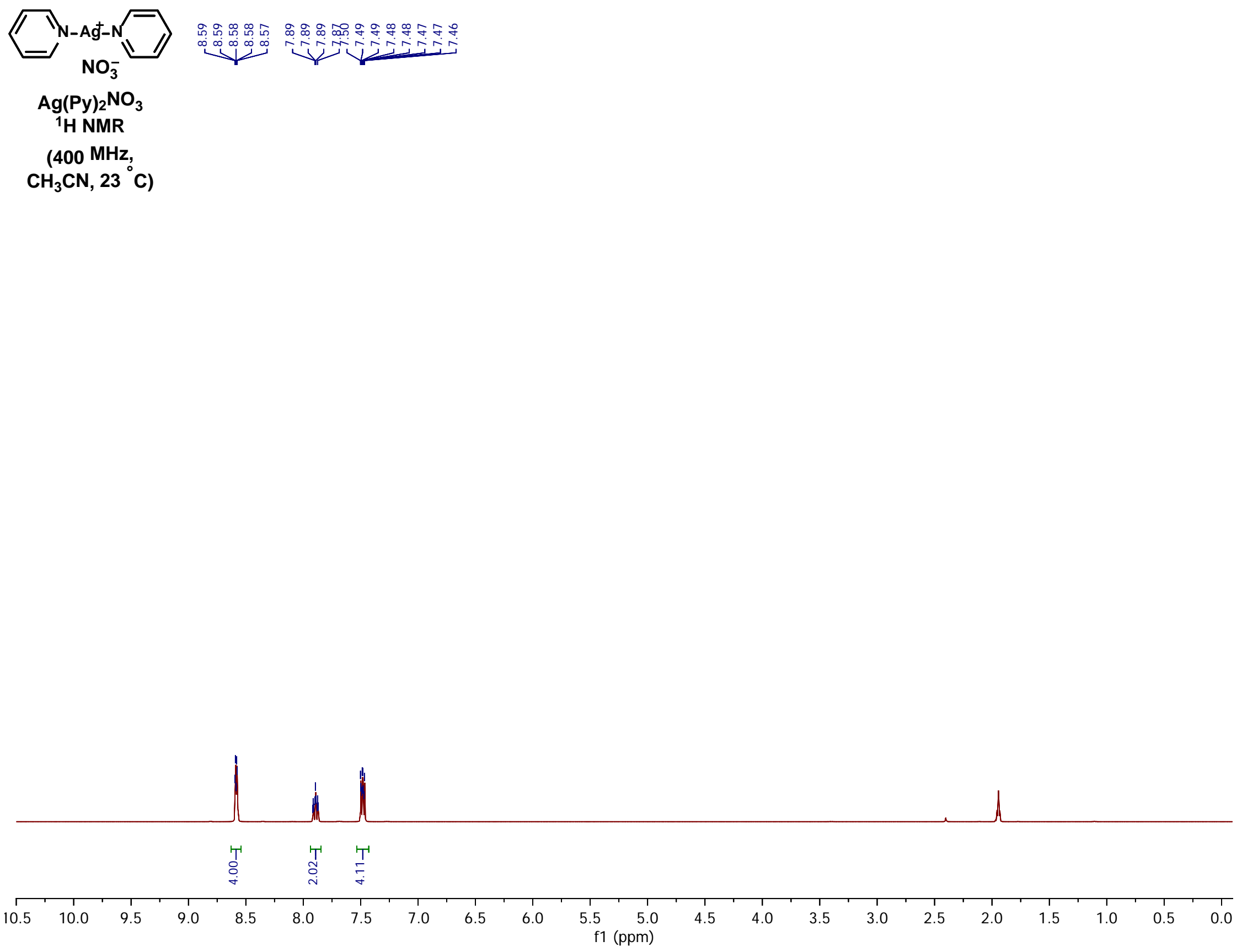




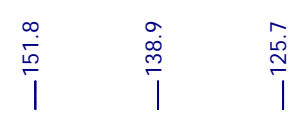

$\mathrm{Ag}(\mathrm{Py})_{2} \mathrm{NO}_{3}$

${ }^{13} \mathrm{C}\left\{{ }^{1} \mathrm{H}\right\}$ NMR

$\left(100 \mathrm{MHz}, \mathrm{CH}_{3} \mathrm{CN}, 23^{\circ} \mathrm{C}\right)$

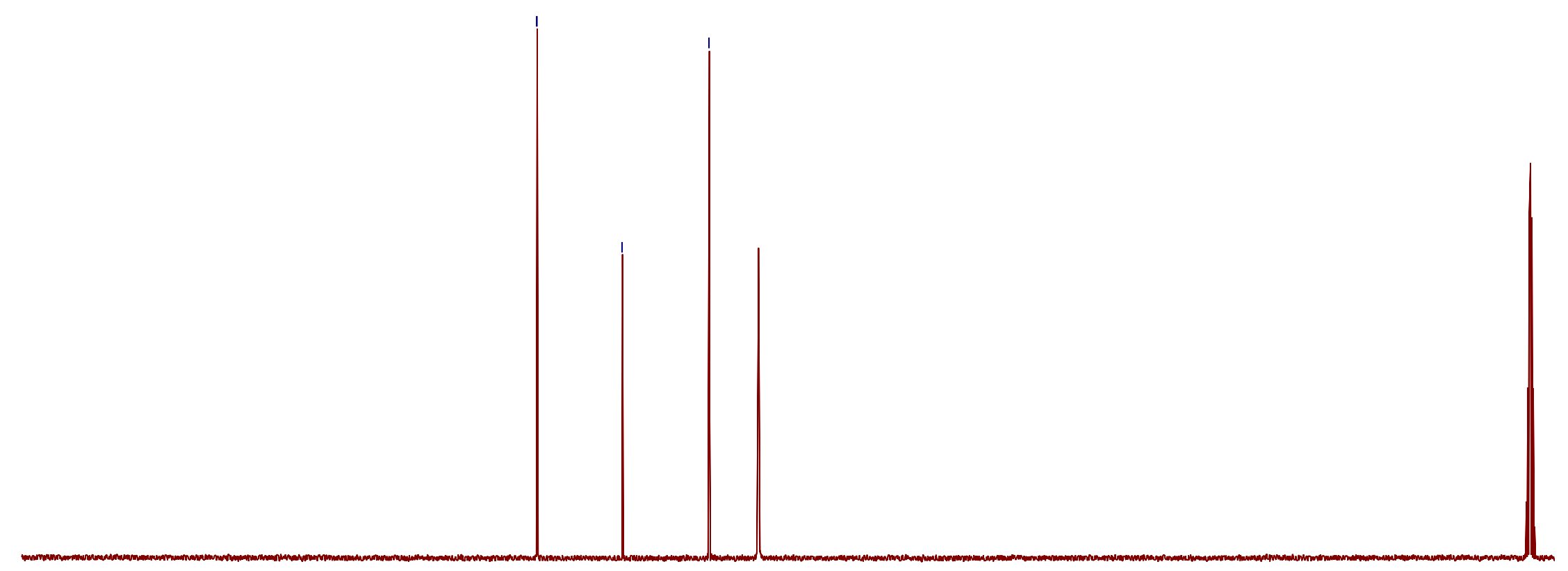

$\begin{array}{rrrrrrrrrrrrrrrrrrrrrrrrrrrrr}230 & 220 & 210 & 200 & 190 & 180 & 170 & 160 & 150 & 140 & 130 & 120 & 110 & 100 & 90 & 80 & 70 & 60 & 50 & 40 & 30 & 20 & 10 & 0\end{array}$





Ag(4-tBuPy) ${ }_{2} \mathrm{NO}_{3}$

${ }^{1} \mathrm{H}$ NMR

(400 $\mathrm{MHz}, \mathrm{CH}_{3} \mathrm{CN}, 23^{\circ} \mathrm{C}$ )





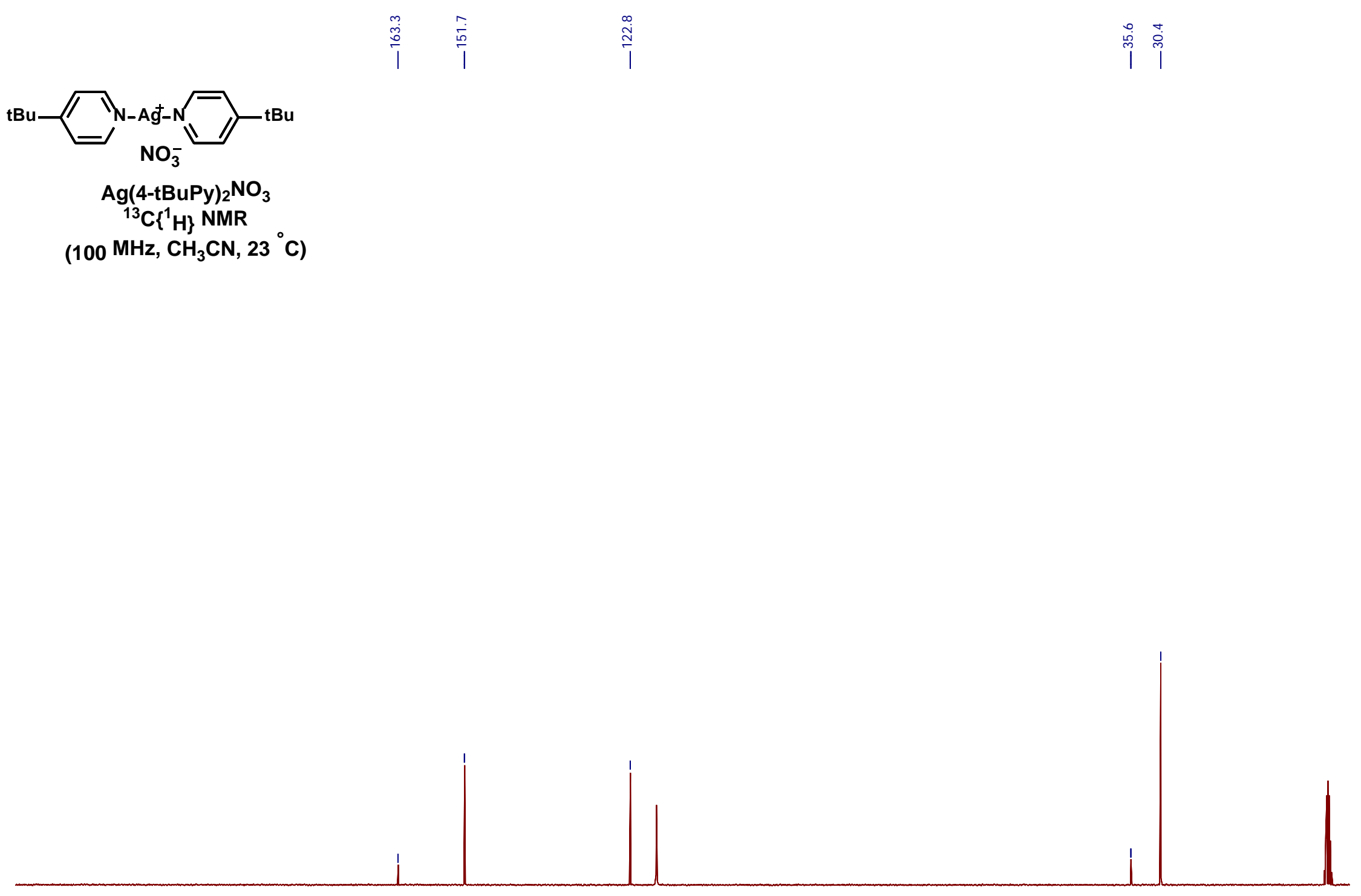

$\begin{array}{rrrrrrrrrrrrrrrrrrrrrrrrrrr}230 & 220 & 210 & 200 & 190 & 180 & 170 & 160 & 150 & 140 & 130 & 120 & 110 & 100 & 90 & 80 & 70 & 60 & 50 & 40 & 30 & 20 & 10 & 0\end{array}$




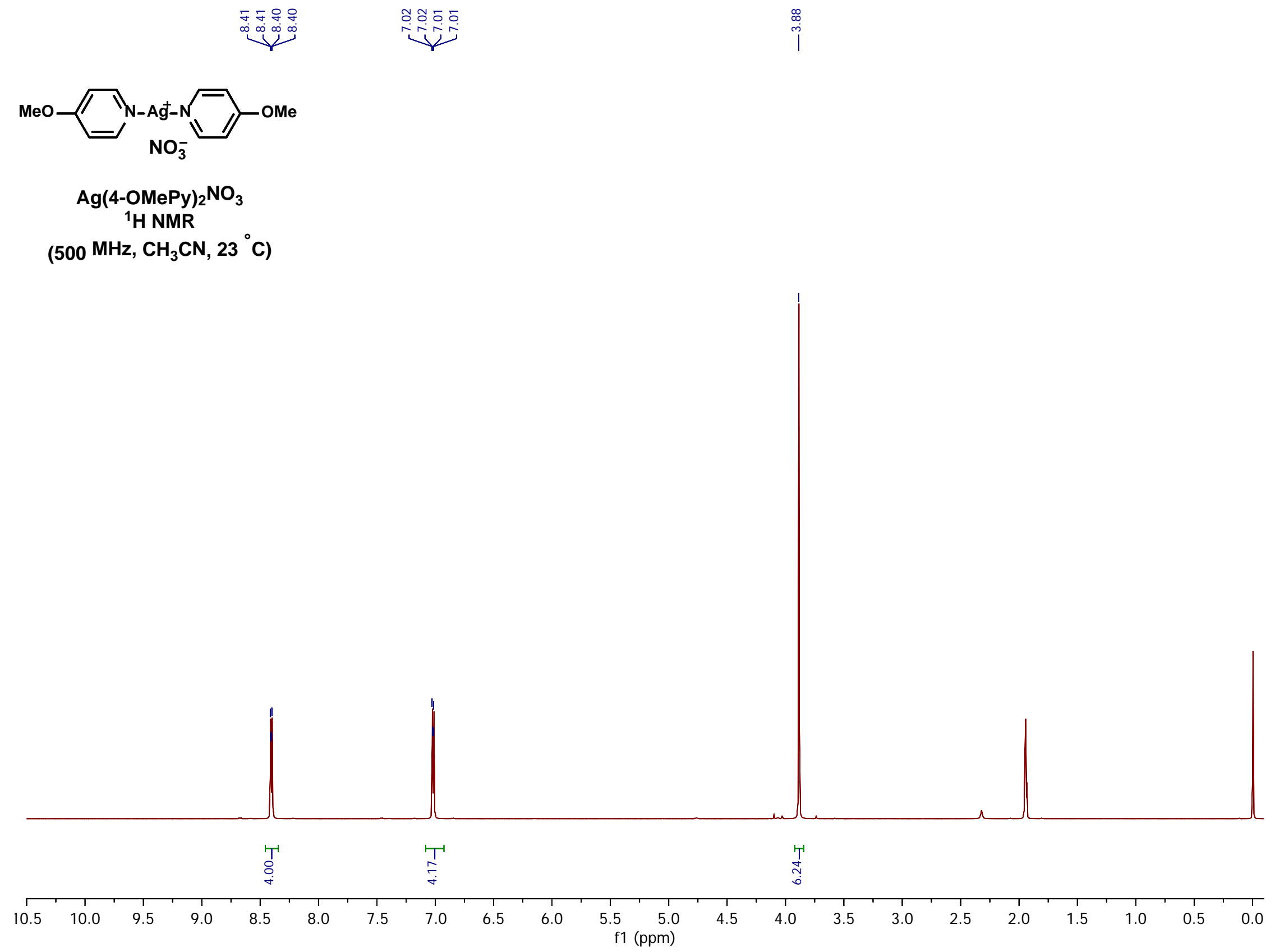





$\begin{array}{rllllllllllllllllllllllllllllll}230 & 220 & 210 & 200 & 190 & 180 & 170 & 160 & 150 & 140 & 130 & 120 & 110 & 100 & 90 & 80 & 70 & 60 & 50 & 40 & 30 & 20 & 10 & 0\end{array}$


تصن

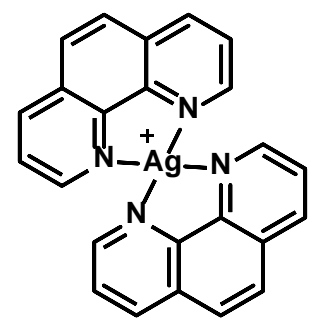

$\mathrm{NO}_{3}^{-}$

$\mathrm{Ag}$ (phen) ${ }_{2} \mathrm{NO}_{3}$

${ }^{1} \mathrm{H}$ NMR

(500 MHz

$\mathrm{CH}_{3} \mathrm{CN}, 23^{\circ} \mathrm{C}$ )










$\mathrm{Ag}(\text { phen })_{2} \mathrm{NO}_{3}$

${ }^{13} \mathrm{C}\left\{{ }^{1} \mathrm{H}\right\}$ NMR

(125 MHz,

$\left.\mathrm{CH}_{3} \mathrm{CN}, 23^{\circ} \mathrm{C}\right)$

$\begin{array}{llllllllllllllllllllllllllll}230 & 220 & 210 & 200 & 190 & 180 & 170 & 160 & 150 & 140 & 130 & 120 & 110 & 100 & 90 & 80 & 70 & 60 & 50 & 40 & 30 & 20 & 10 & 0\end{array}$




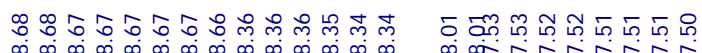
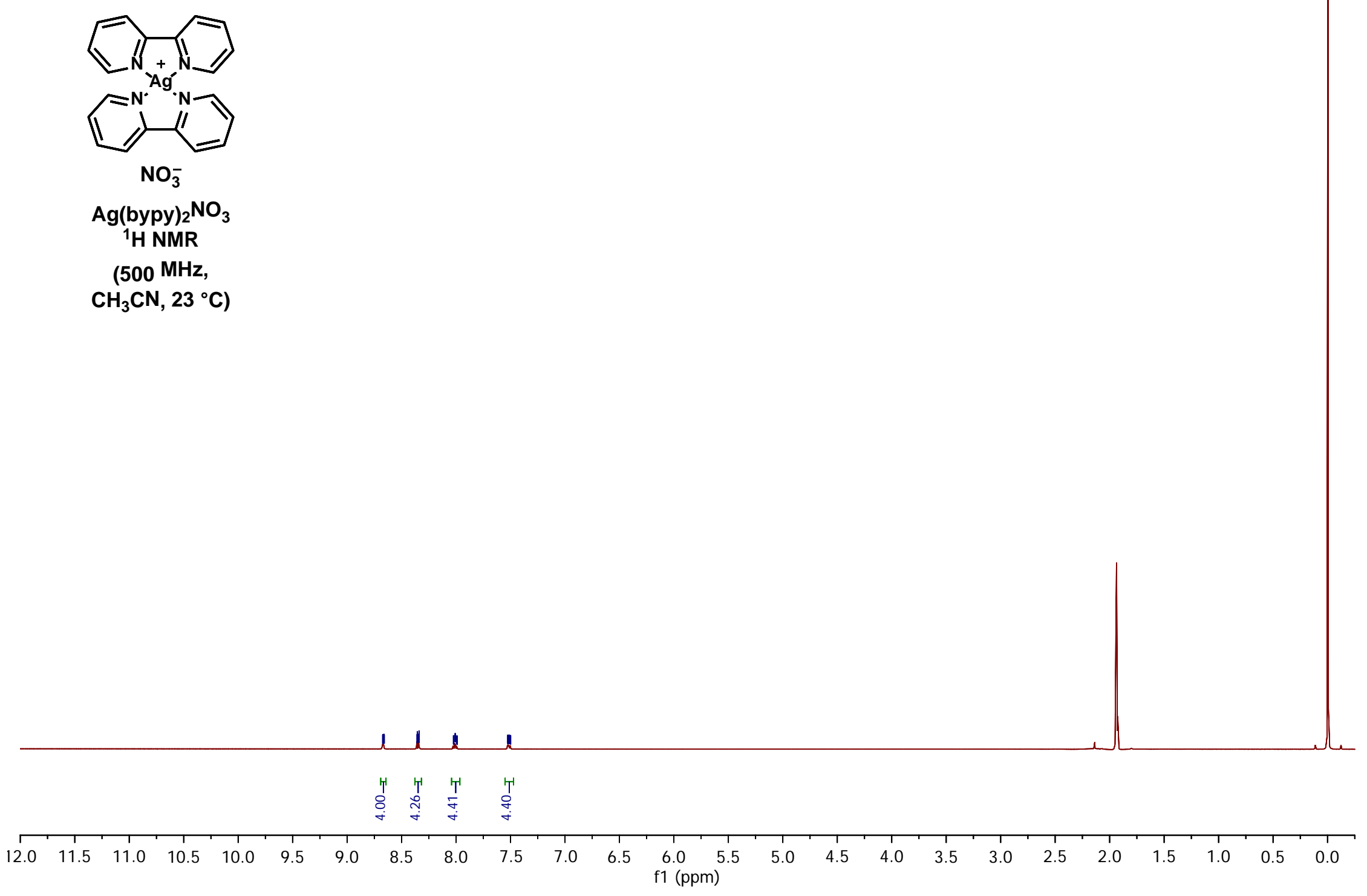


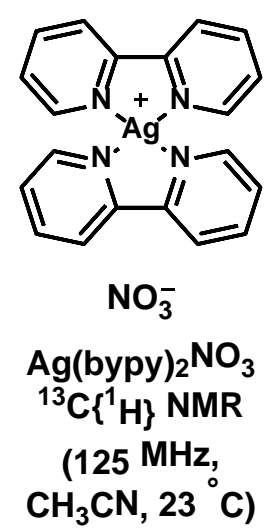

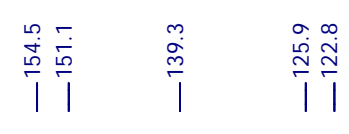

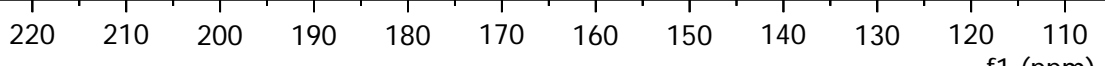
f1 (ppm) 


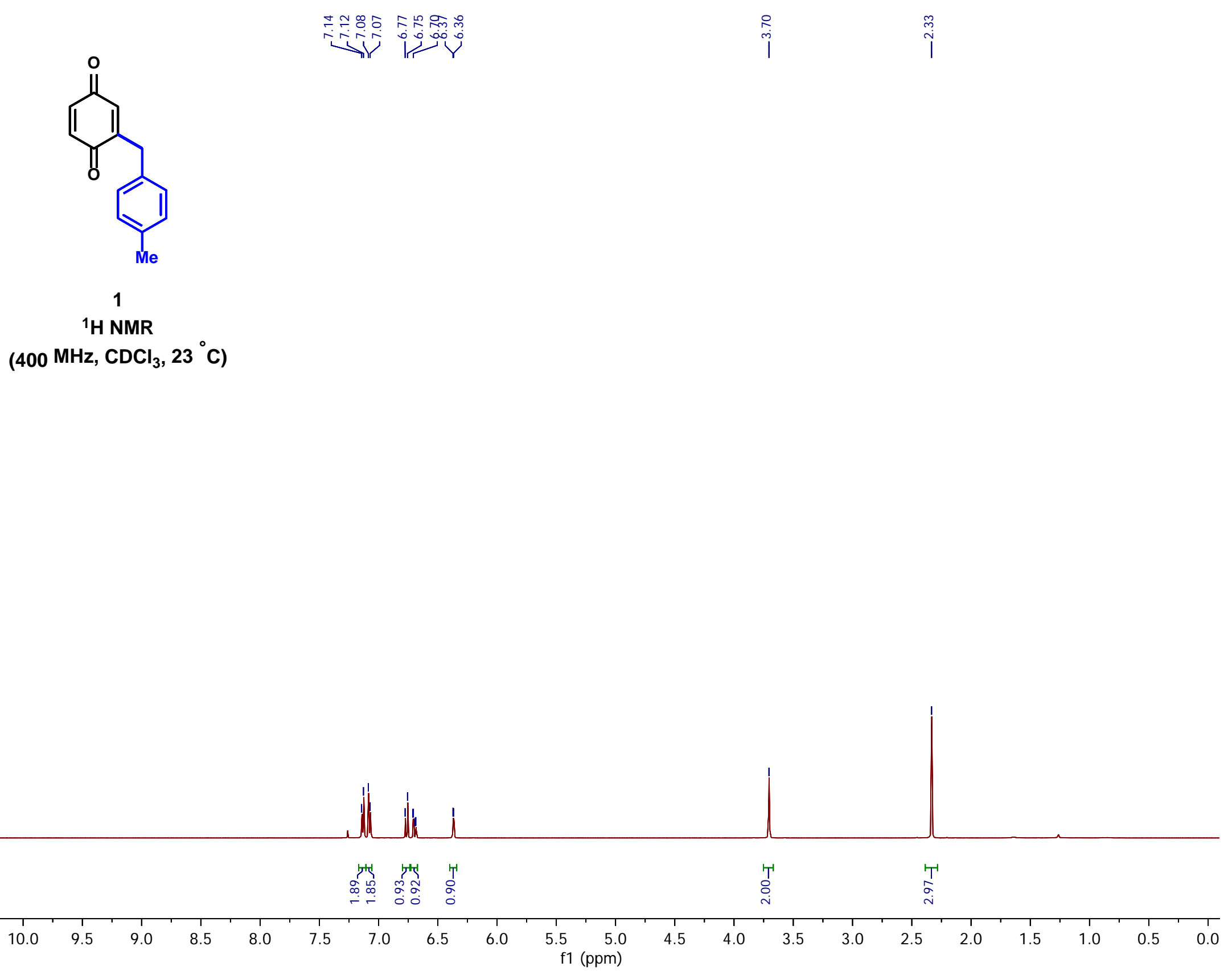


S21

గ্c

高

$\stackrel{9}{\dot{m}} \stackrel{\stackrel{N}{+}}{1}$<smiles>Cc1ccc(CC2=CC(=O)C=CC2=O)cc1</smiles>
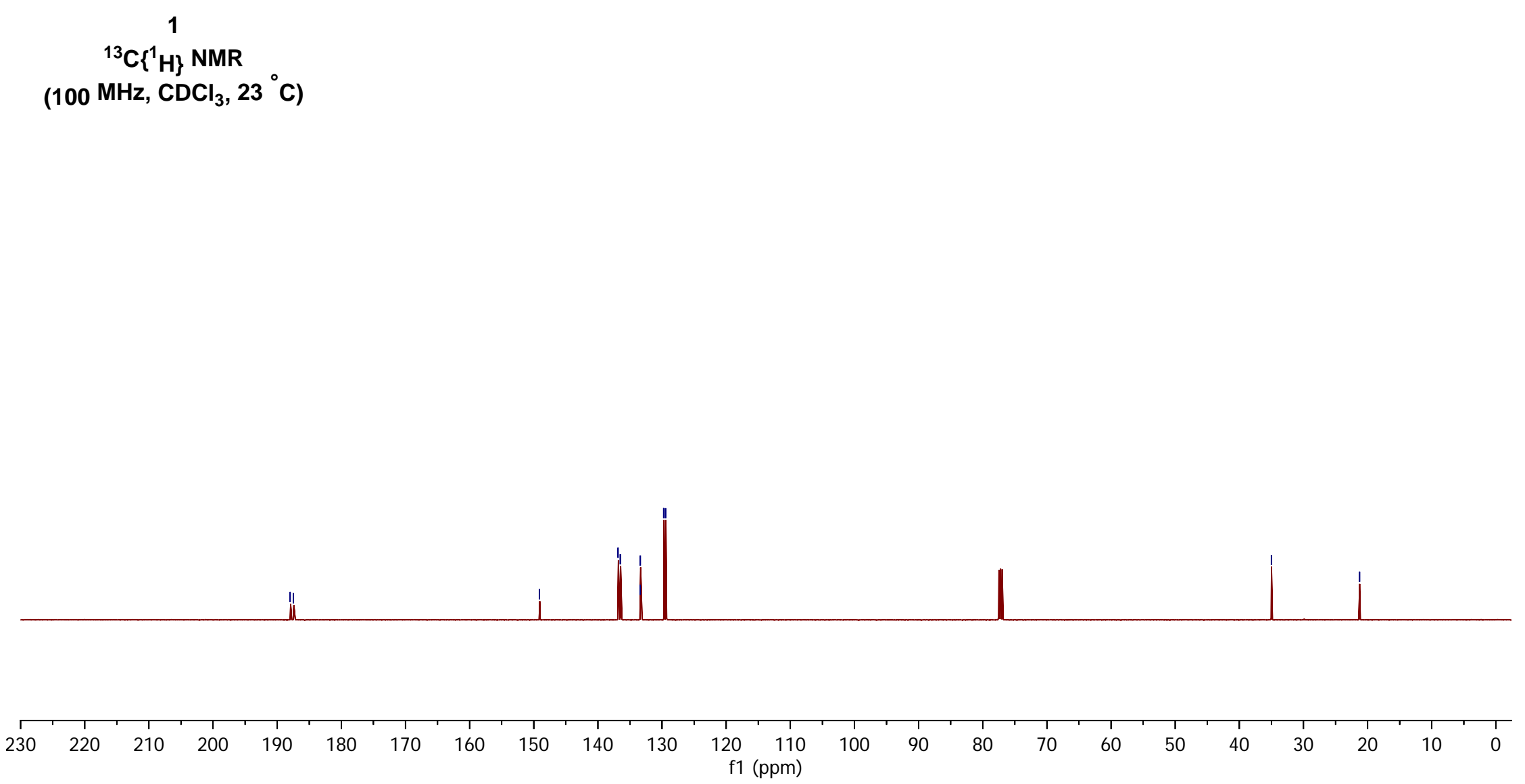


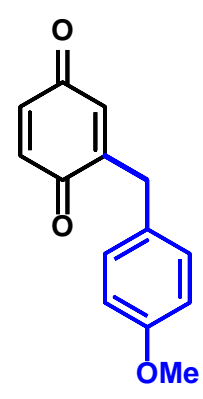

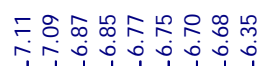

1

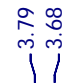

2

${ }^{1} \mathrm{H}$ NMR

(500 MHz, $\mathrm{CDCl}_{3}, 23^{\circ} \mathrm{C}$ )

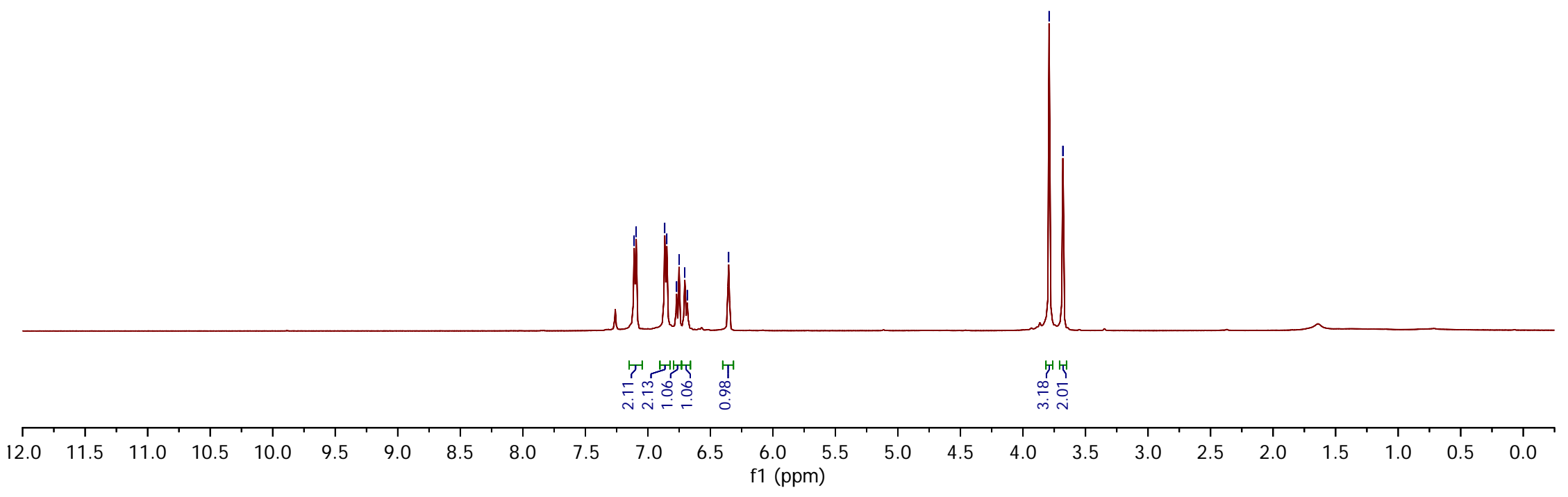



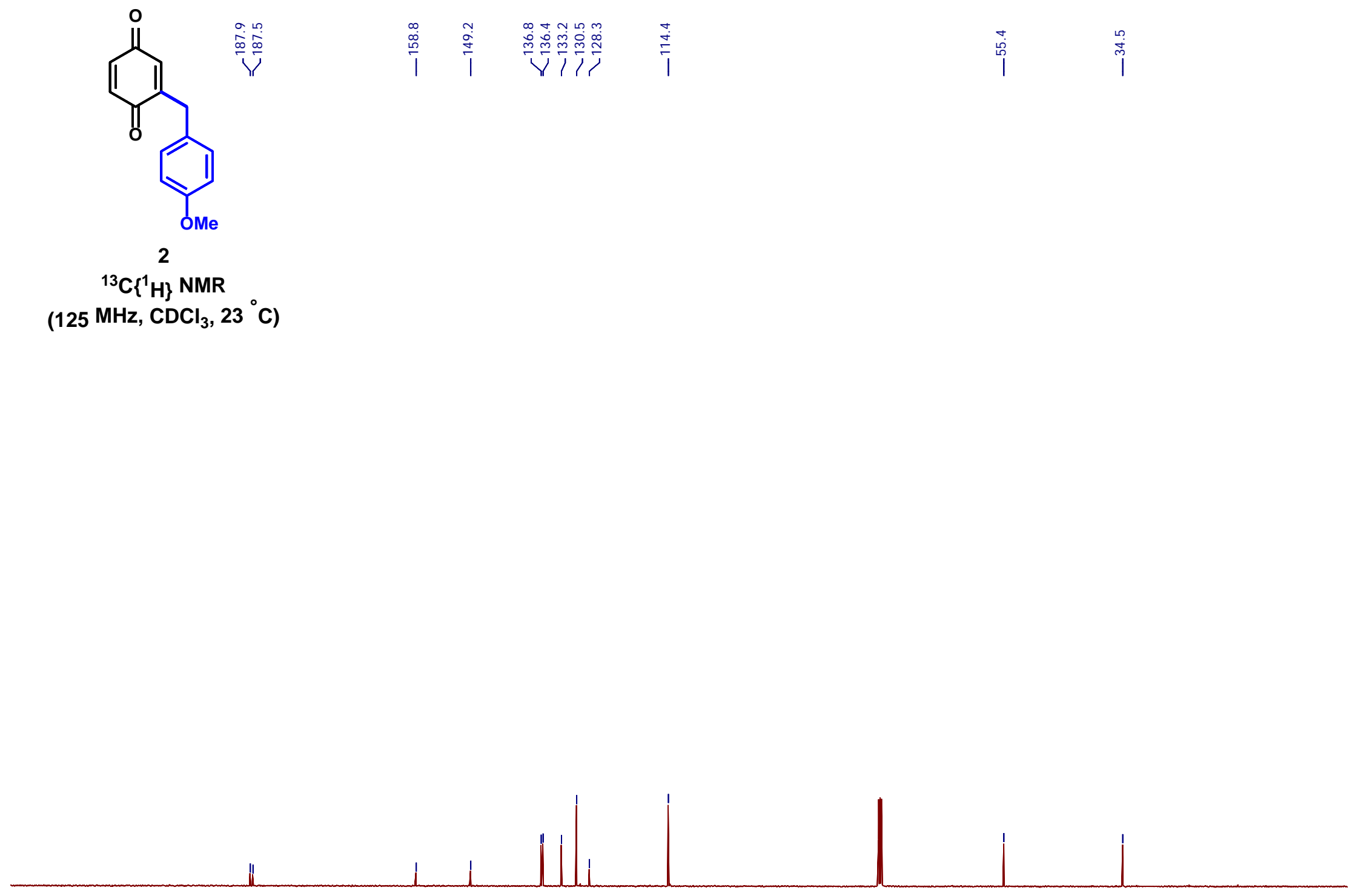

\begin{tabular}{rrrrrrrrrrrrrrrrrrrrrrrrrrrrrrrr}
\hline 230 & 220 & 210 & 200 & 190 & 180 & 170 & 160 & 150 & 140 & 130 & 120 & 110 & 100 & 90 & 80 & 70 & 60 & 50 & 40 & 30 & 20 & 10 & 0
\end{tabular}




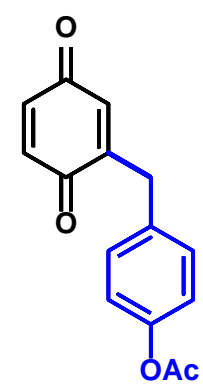

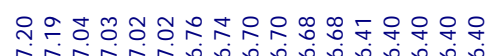

年

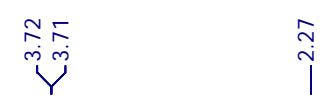

3

${ }^{1} \mathrm{H}$ NMR

(500 MHz, $\mathrm{CDCl}_{3}, 23^{\circ} \mathrm{C}$ )

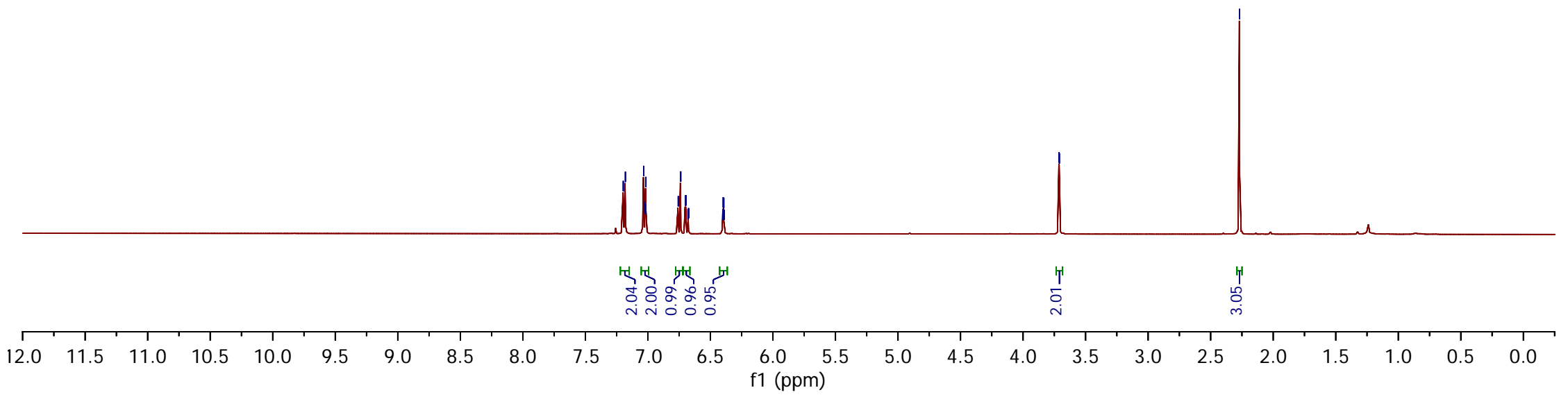




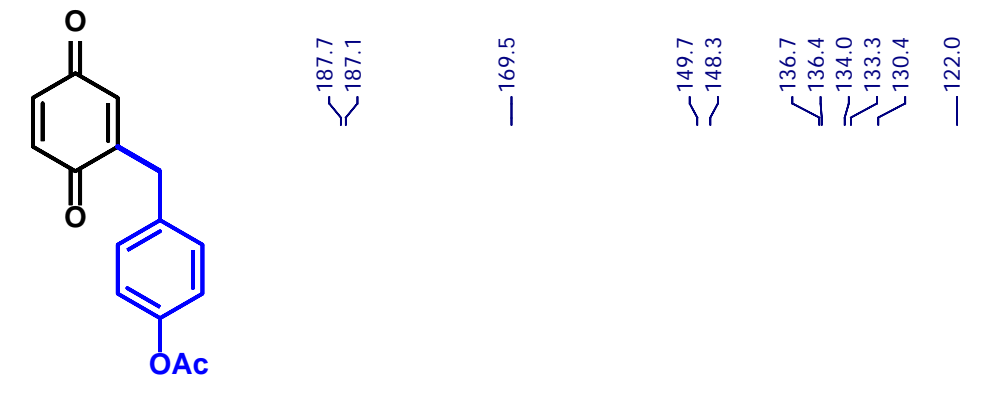

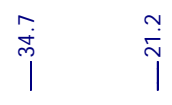

${ }^{13} \mathrm{C}\left\{{ }^{1} \mathrm{H}\right\}$ NMR

$\left(125 \mathrm{MHz}, \mathrm{CDCl}_{3}, 23^{\circ} \mathrm{C}\right)$

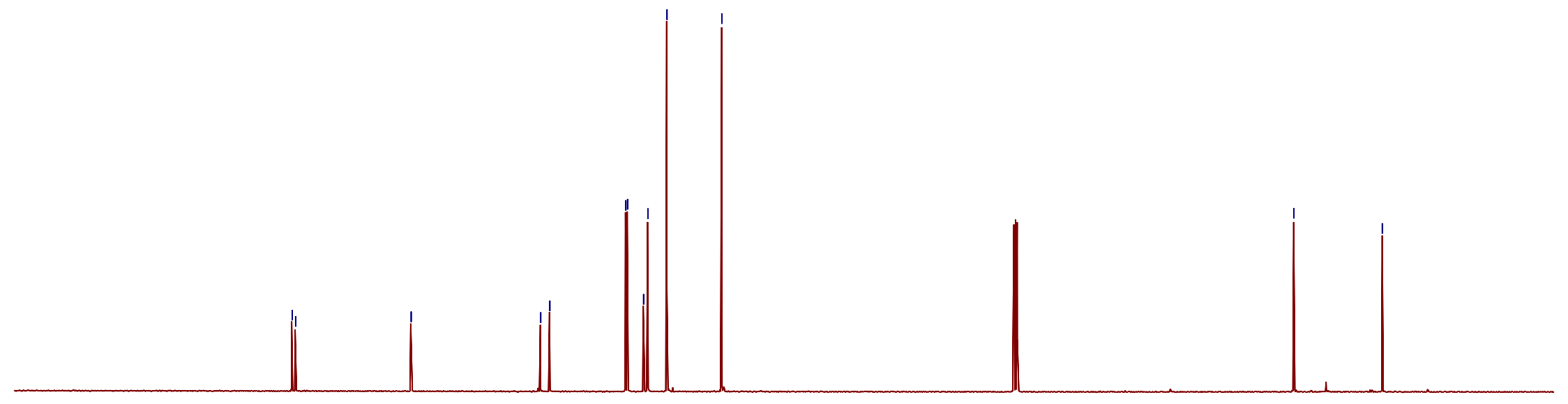

$\begin{array}{rrrrrrrrrrrrrrrrrrrrrrrrrr} & 230 & 220 & 210 & 200 & 190 & 180 & 170 & 160 & 150 & 140 & 130 & 120 & 110 & 100 & 90 & 80 & 70 & 60 & 50 & 40 & 30 & 20 & 10 & 0 & \end{array}$ 
S26<smiles>N#Cc1ccc(CC2=CC(=O)C=CC2=O)cc1</smiles>

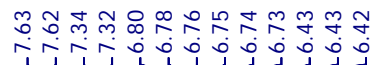

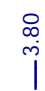
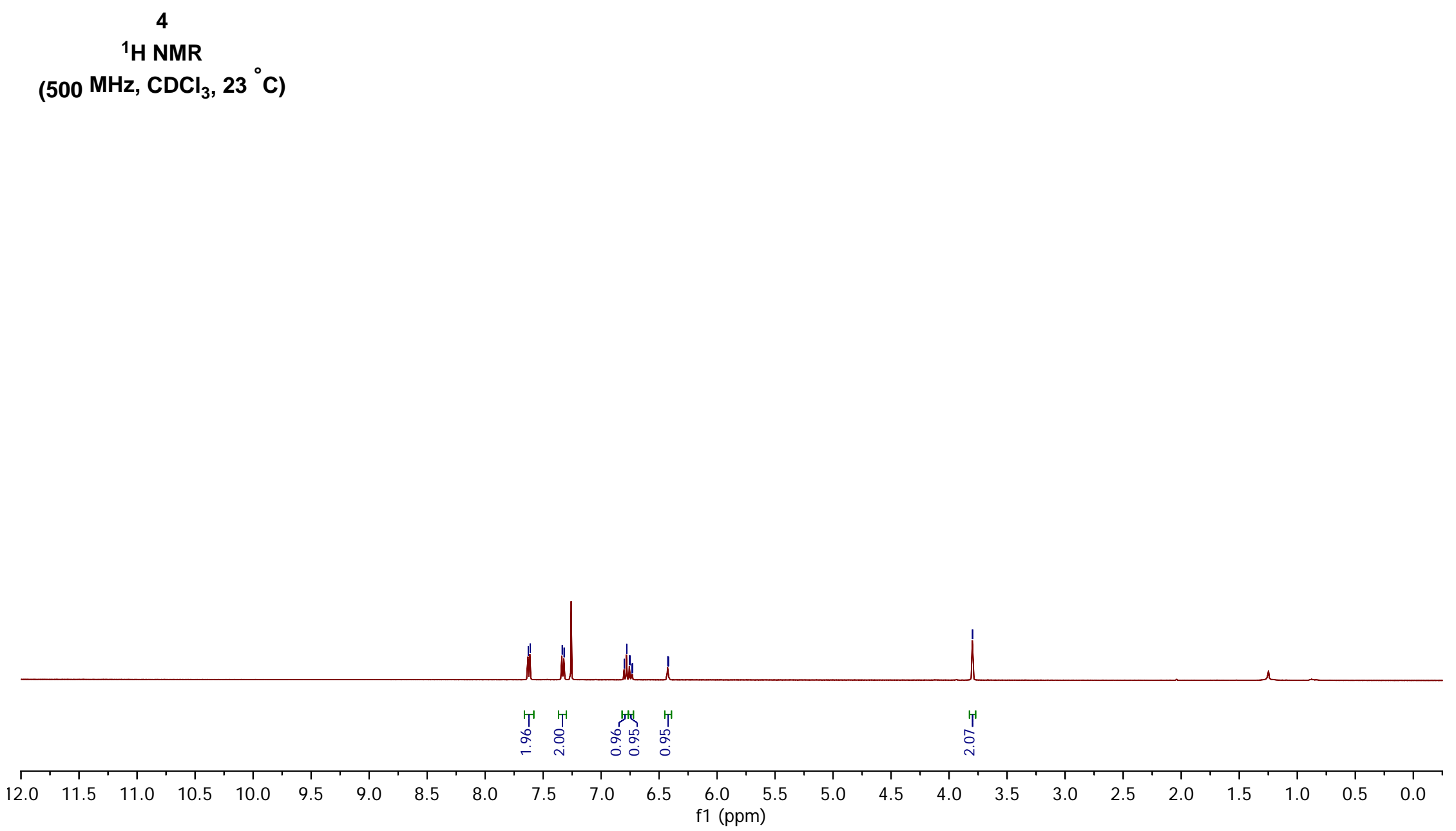


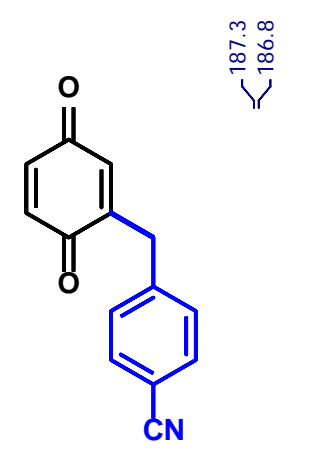

我

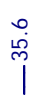

${ }^{13} \mathrm{C}\left\{{ }^{1} \mathrm{H}\right\}$ NMR

(125 MHz, $\mathrm{CDCl}_{3}, 23^{\circ} \mathrm{C}$ )

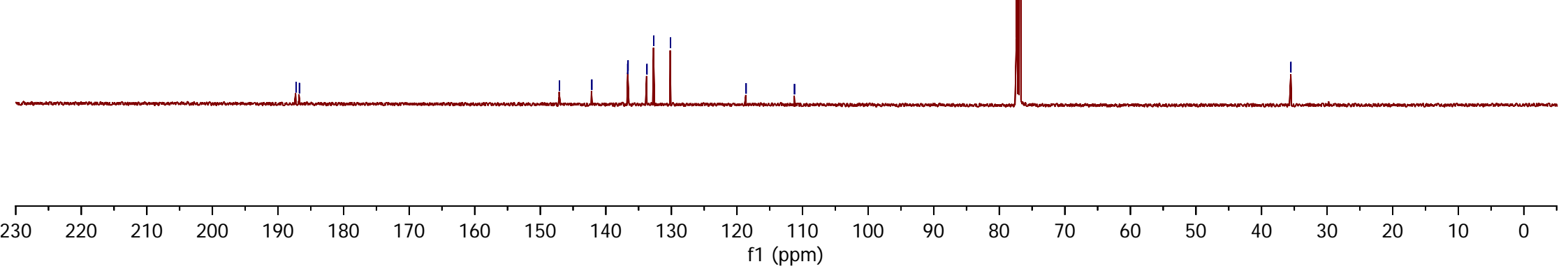




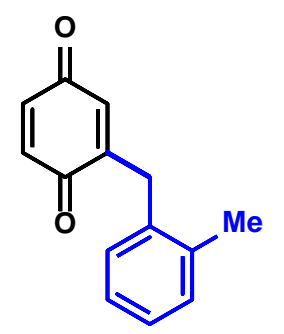

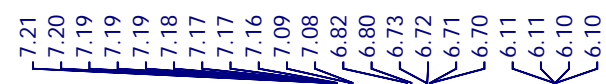

$\underbrace{\stackrel{n}{m}}_{\substack{n \\ m}}$

$\stackrel{i}{i}$

5

${ }^{1} \mathrm{H}$ NMR

(500 MHz, $\mathrm{CDCl}_{3}, 23^{\circ} \mathrm{C}$ )

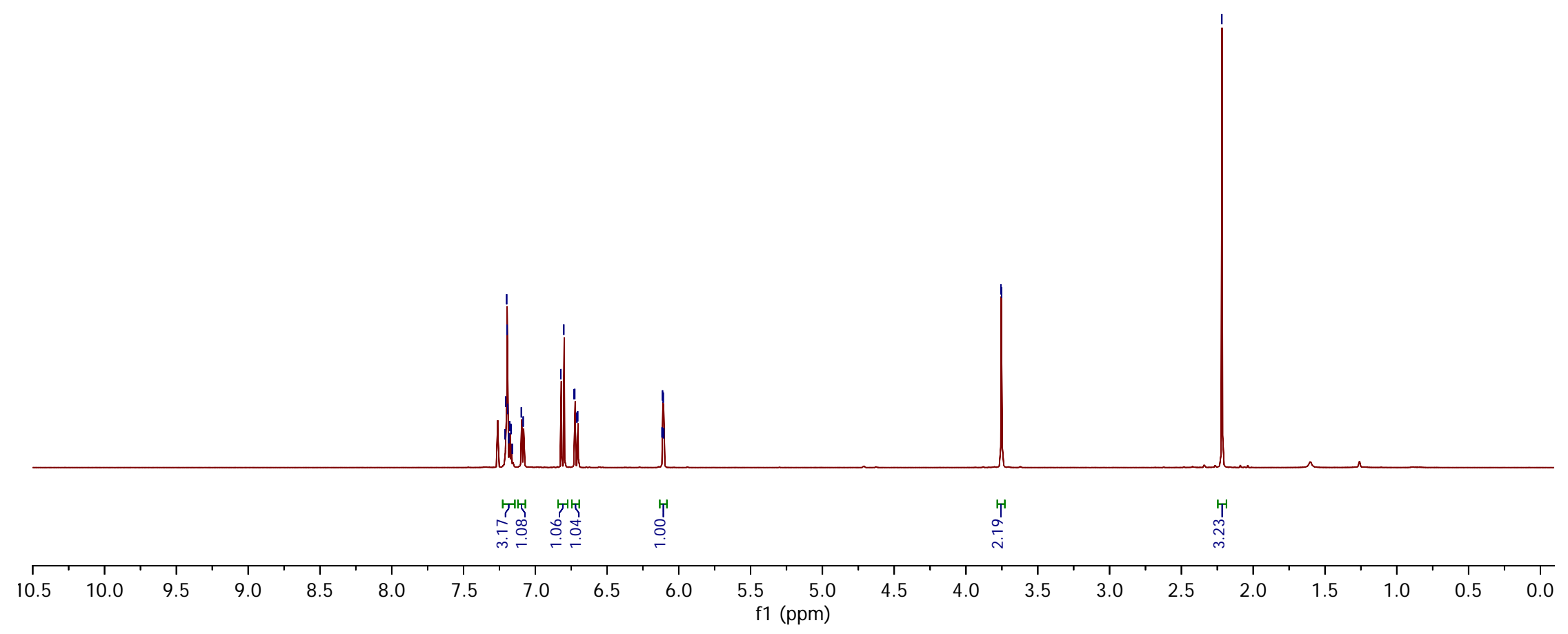


S29<smiles>Cc1ccccc1CC1=CC(=O)C=CC1=O</smiles>

\ִ

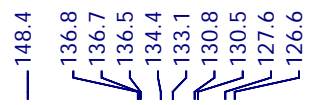

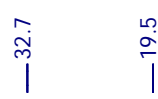

${ }^{13} \mathrm{C}\left\{{ }^{1} \mathrm{H}\right\}$ NMR

(125 $\mathrm{MHz}, \mathrm{CDCl}_{3}, 23^{\circ} \mathrm{C}$ )

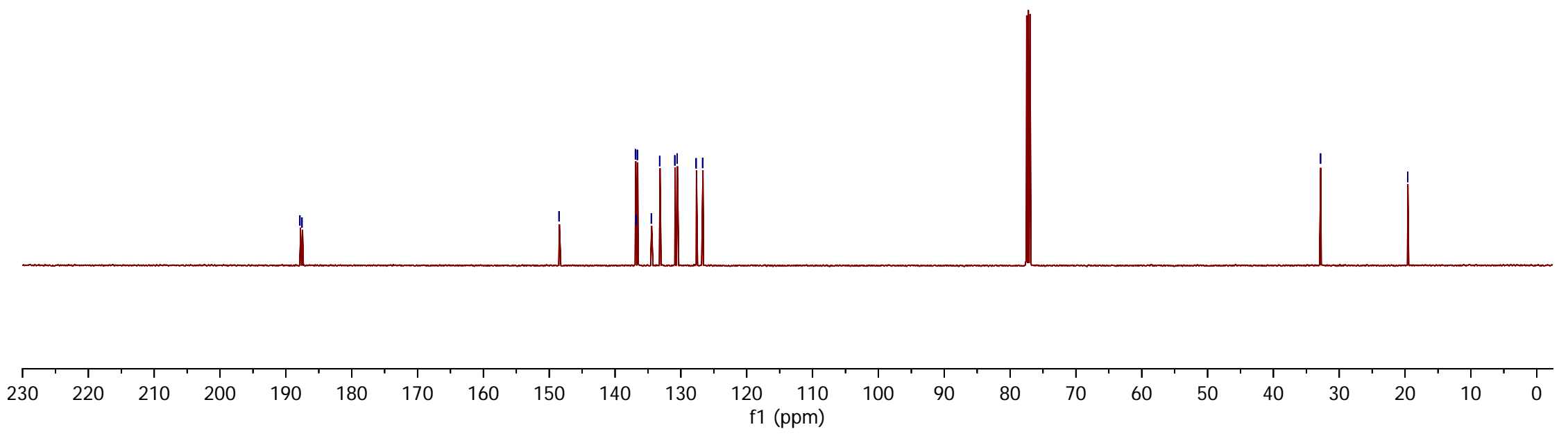




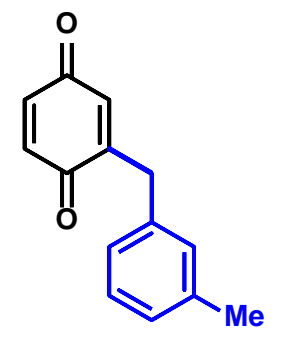

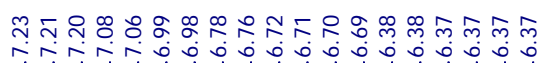

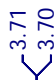

$\stackrel{m}{\sim}$

6

${ }^{1} \mathrm{H}$ NMR

(500 MHz, $\mathrm{CDCl}_{3}, 23^{\circ} \mathrm{C}$ )

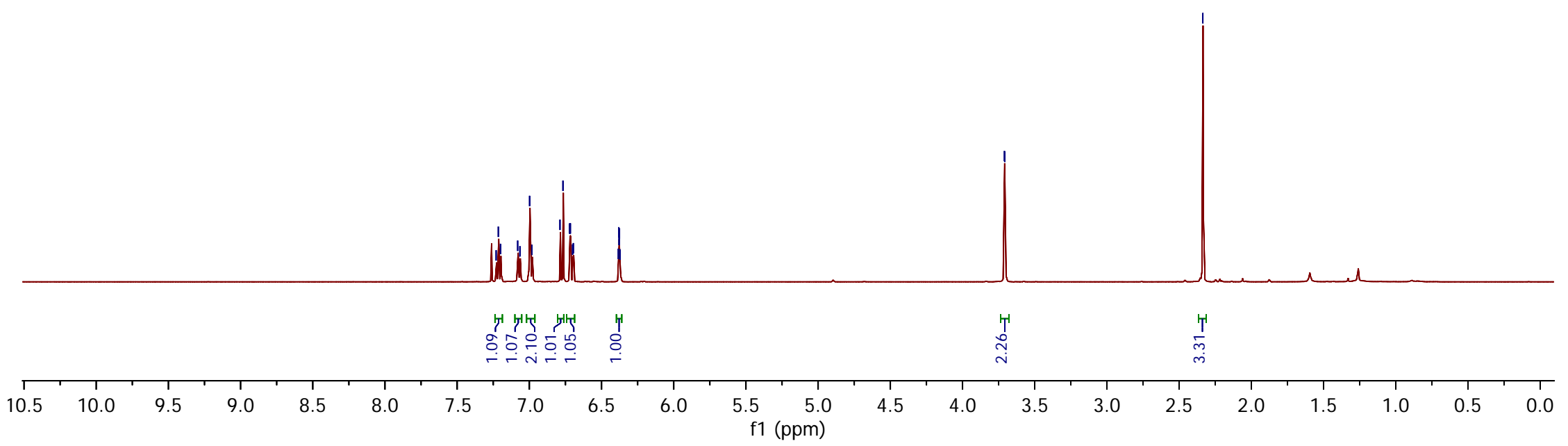




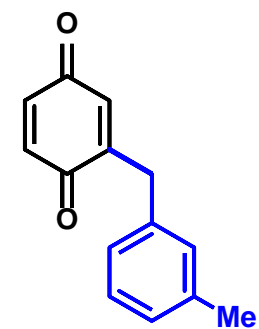

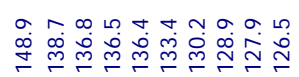

$\stackrel{\sim}{\stackrel{n}{m}} \stackrel{\stackrel{n}{n}}{i}$

6

${ }^{13} \mathrm{C}\left\{{ }^{1} \mathrm{H}\right\}$ NMR

$\left(125 \mathrm{MHz}, \mathrm{CDCl}_{3}, 23^{\circ} \mathrm{C}\right)$

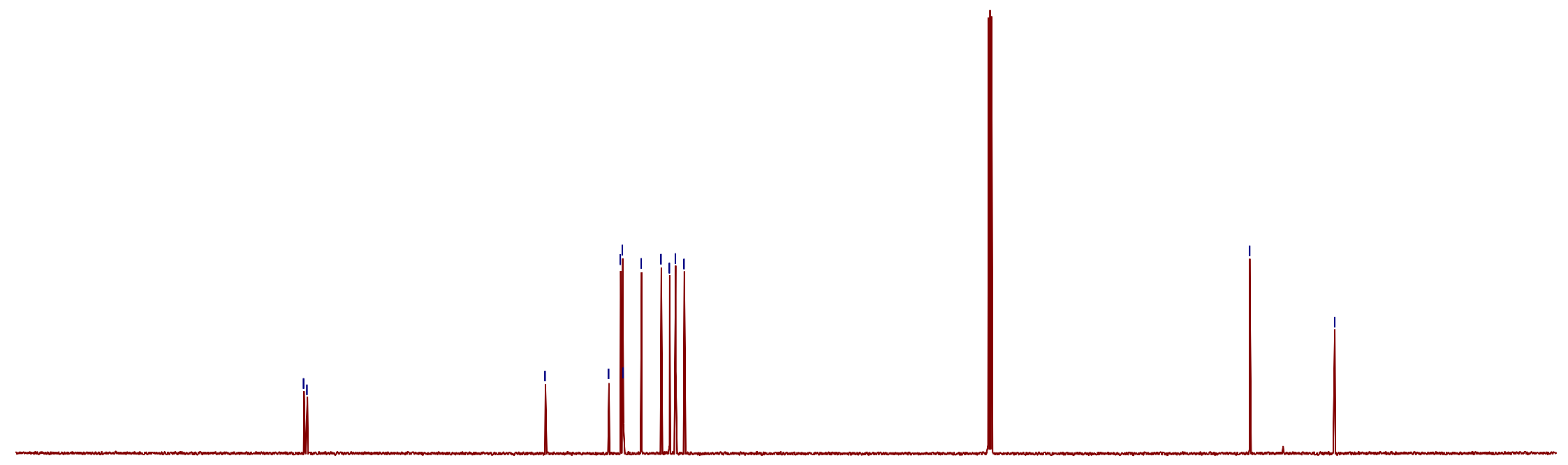

$\begin{array}{lllllllllllllllllllllllllllll}230 & 220 & 210 & 200 & 190 & 180 & 170 & 160 & 150 & 140 & 130 & 120 & 110 & 100 & 90 & 80 & 70 & 60 & 50 & 40 & 30 & 20 & 10 & 0 & -10\end{array}$ 


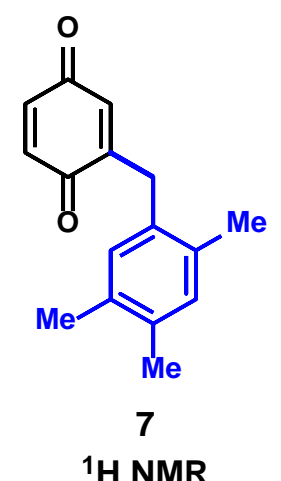

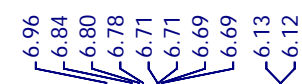

${ }^{1} \mathrm{H}$ NMR

(500 MHz, $\mathrm{CDCl}_{3}, 23^{\circ} \mathrm{C}$ )

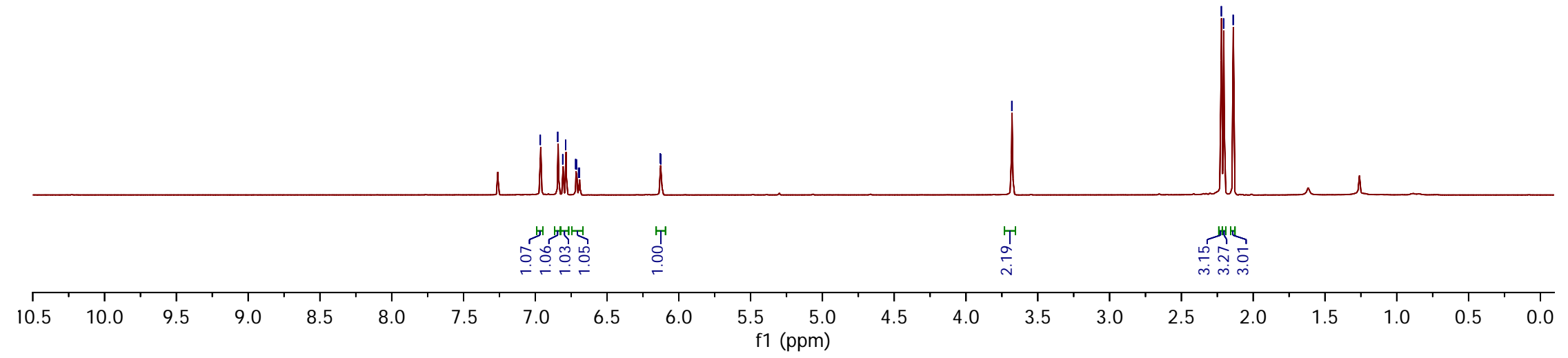




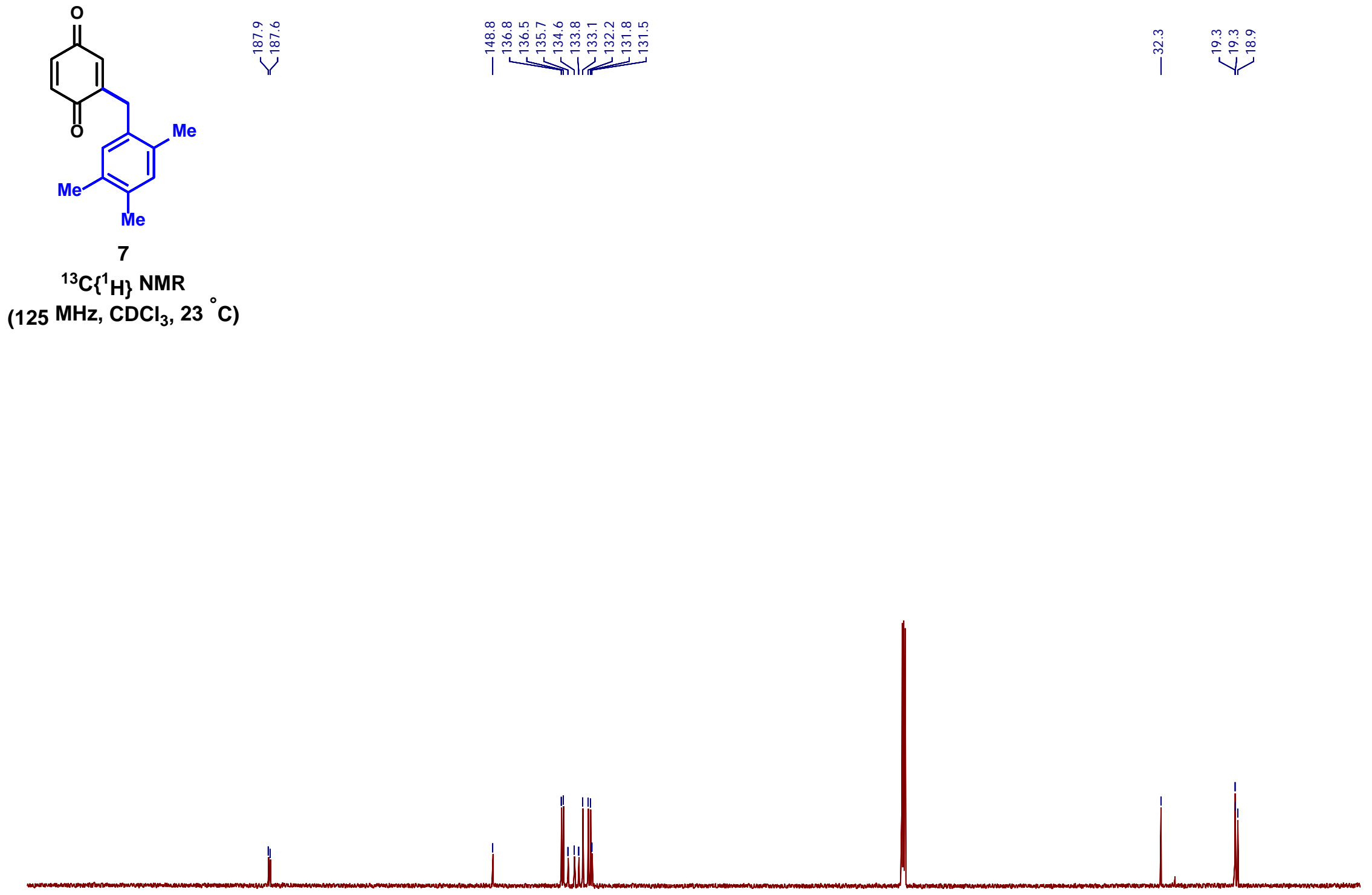

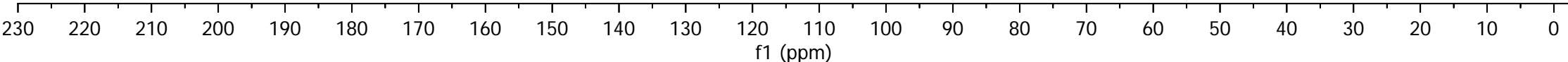




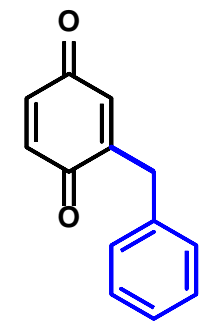

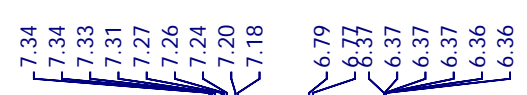



8

${ }^{1} \mathrm{H}$ NMR

(500 MHz, $\mathrm{CDCl}_{3}, 23^{\circ} \mathrm{C}$ )

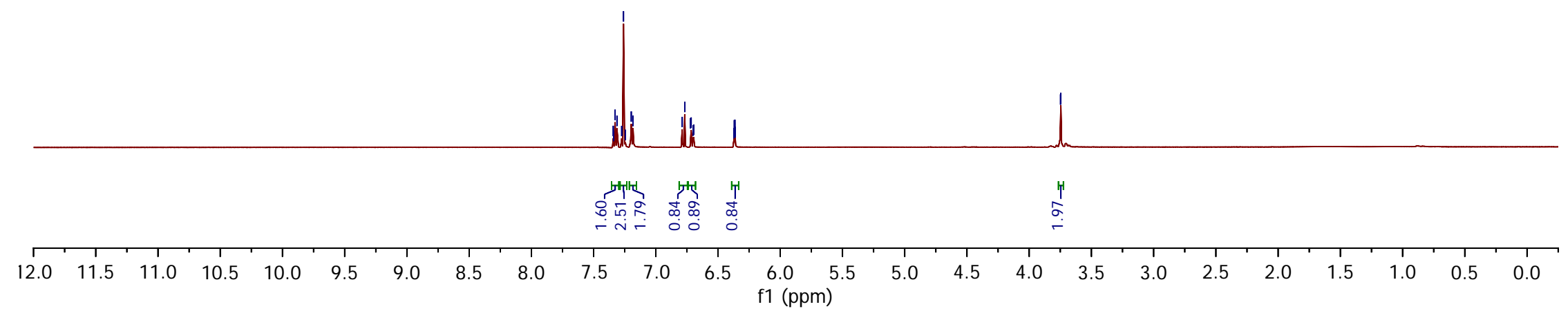




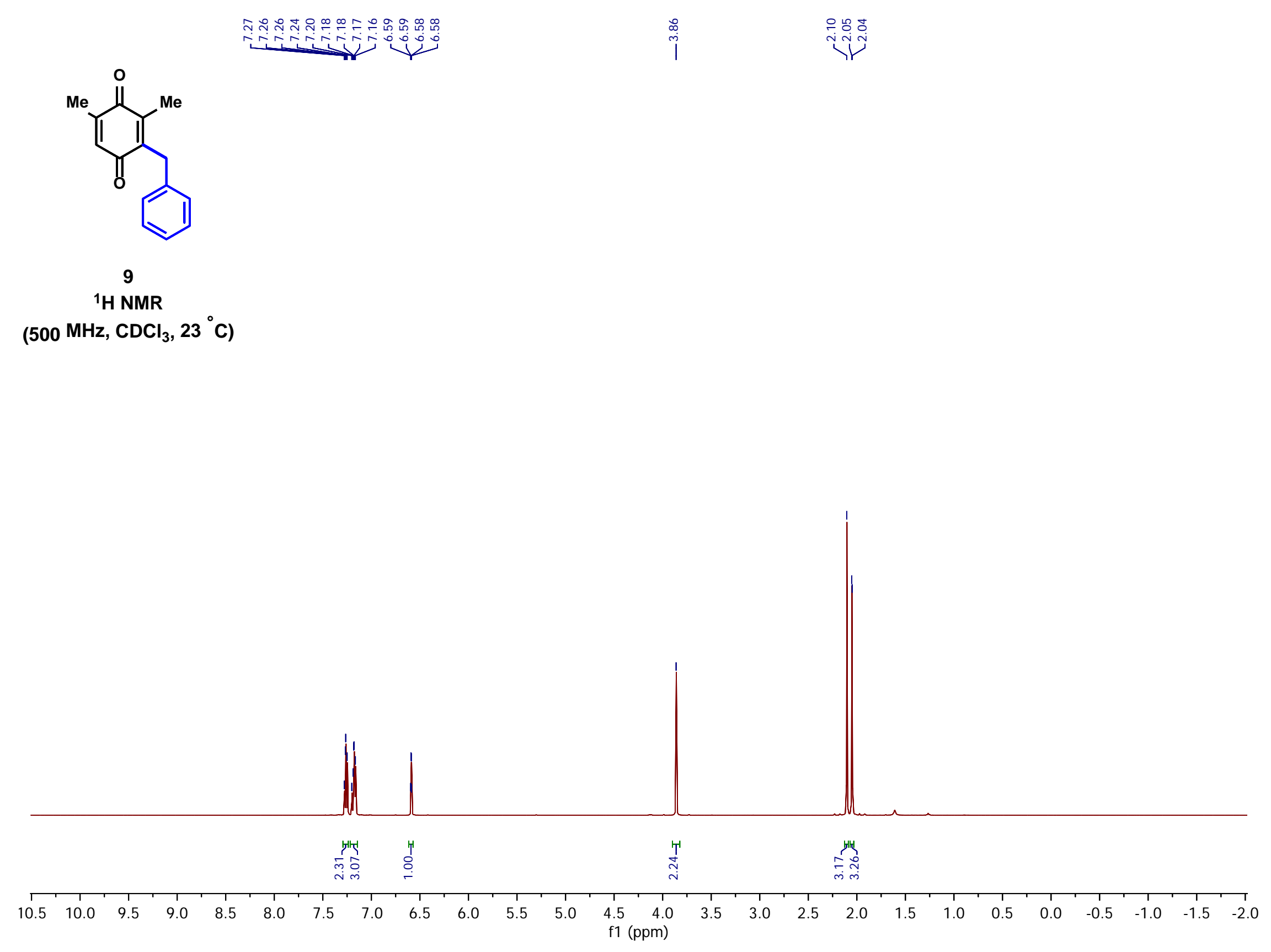


S36

10

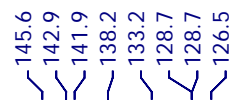

iे

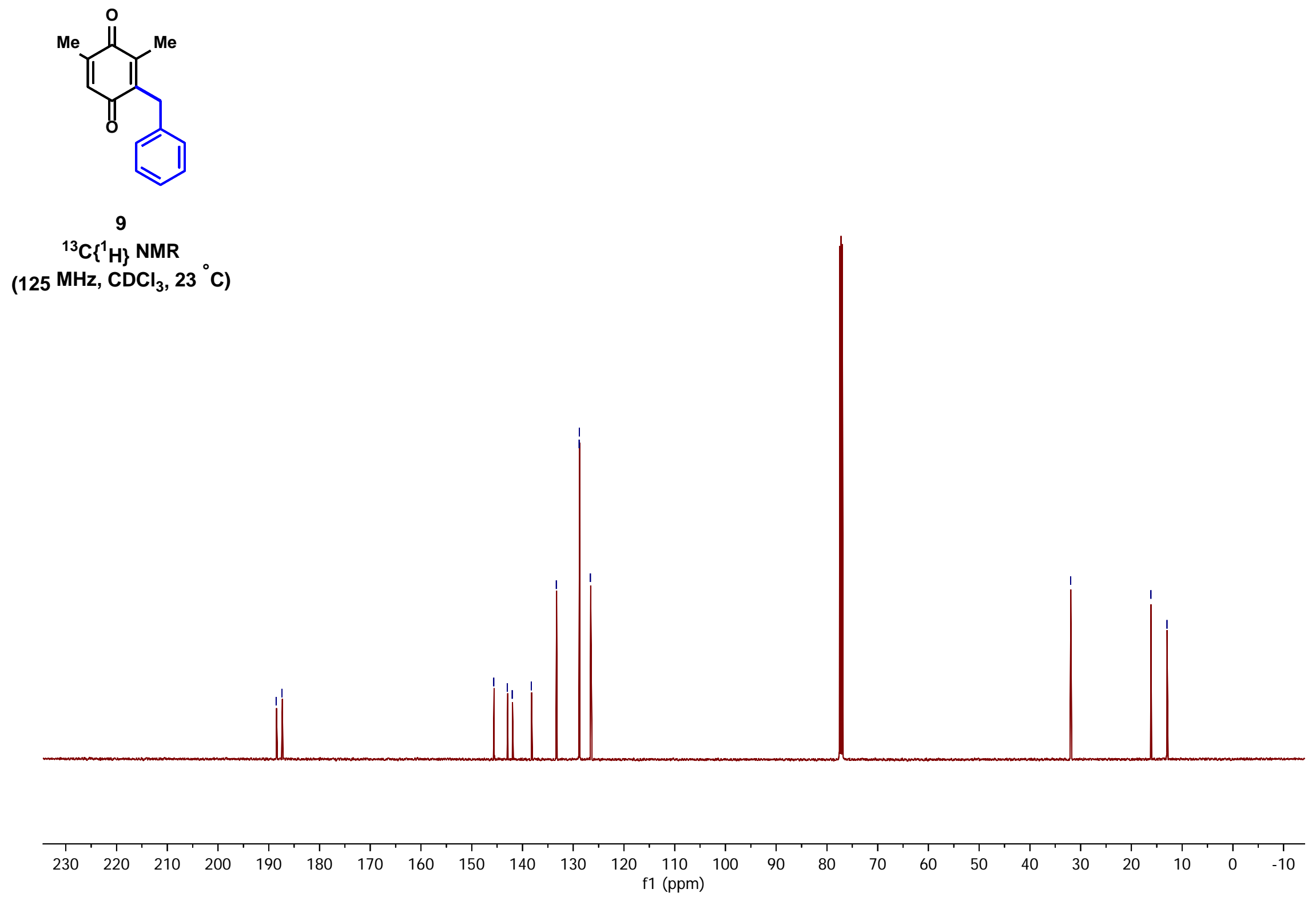




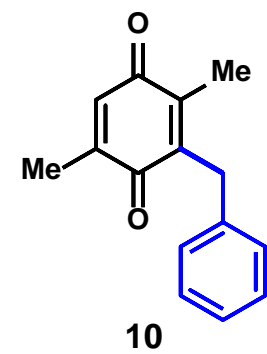

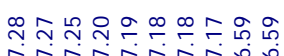

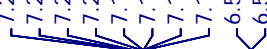

$\stackrel{\infty}{i}$

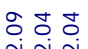

$\sqrt[4]{8.42}$

${ }^{1} \mathrm{H}$ NMR

(500 MHz, $\mathrm{CDCl}_{3}, 23^{\circ} \mathrm{C}$ )

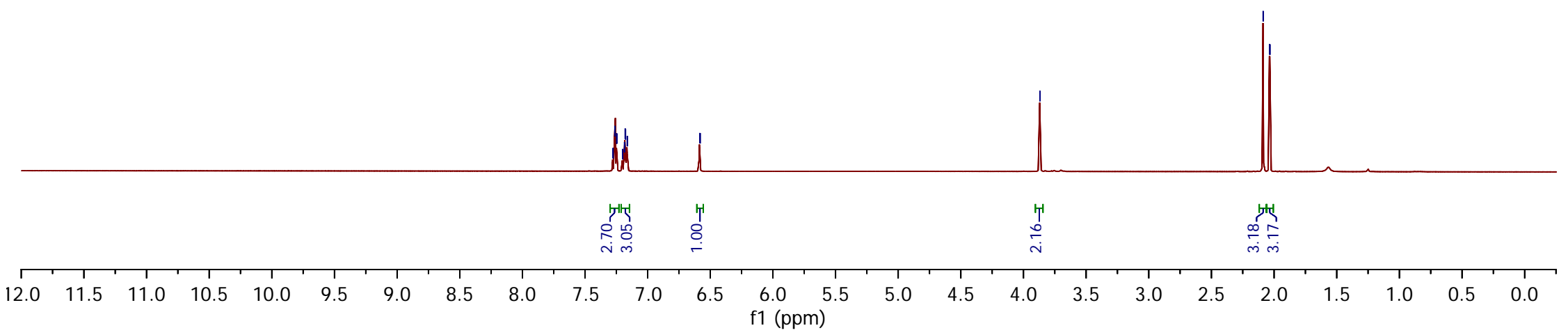




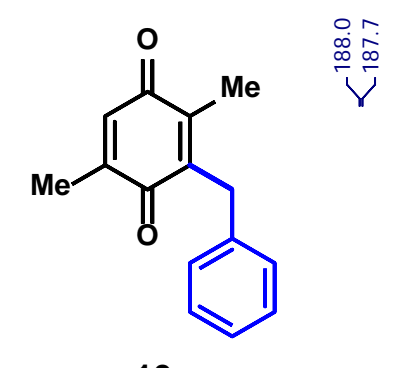

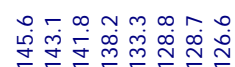

$\stackrel{\substack{\tilde{N} \\ m}}{i}$

10

${ }^{13} \mathrm{C}\left\{{ }^{1} \mathrm{H}\right\}$ NMR

(125 MHz, $\mathrm{CDCl}_{3}, 23^{\circ} \mathrm{C}$ )

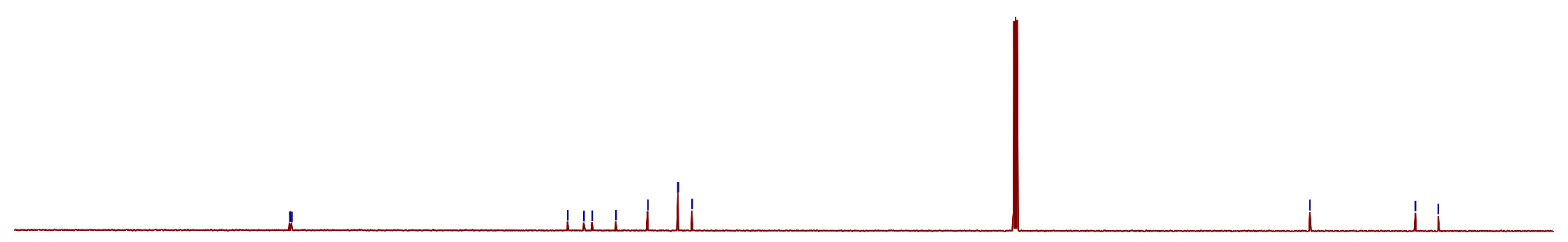




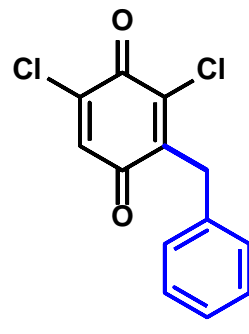

ำ

赵

11

${ }^{1} \mathrm{H}$ NMR

(400 MHz, $\mathrm{CDCl}_{3}, 23^{\circ} \mathrm{C}$ )

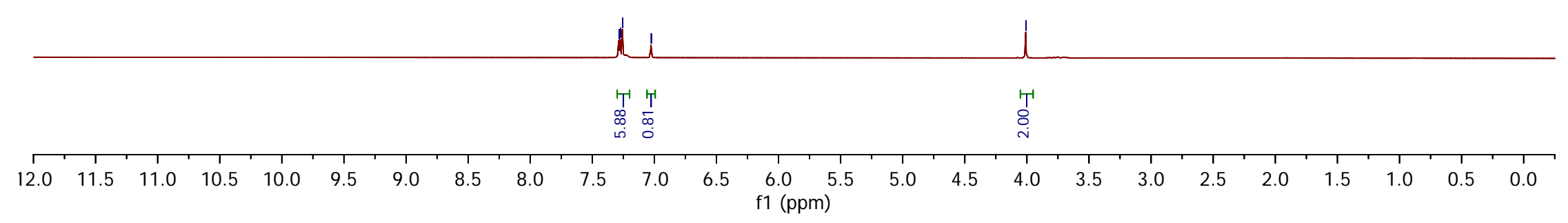




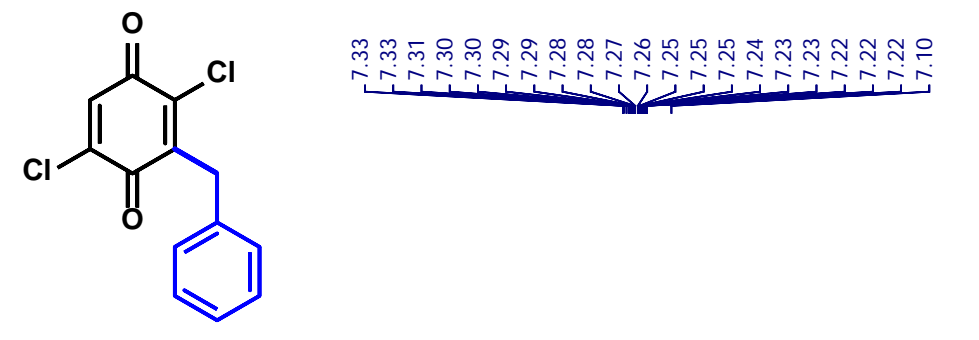

12

${ }^{1}$ H NMR

(500 MHz, $\mathrm{CDCl}_{3}, 23^{\circ} \mathrm{C}$ )

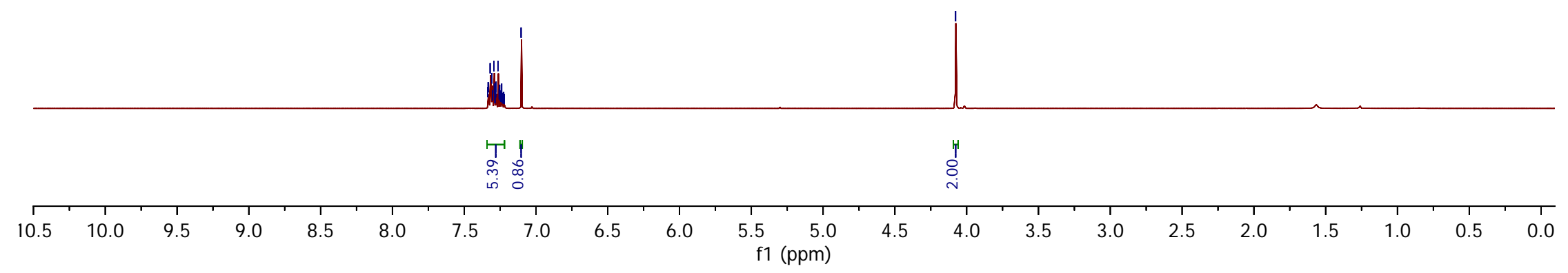




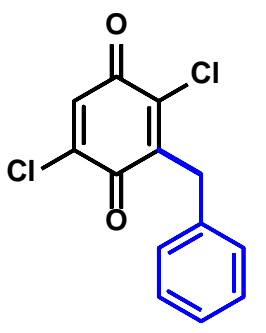

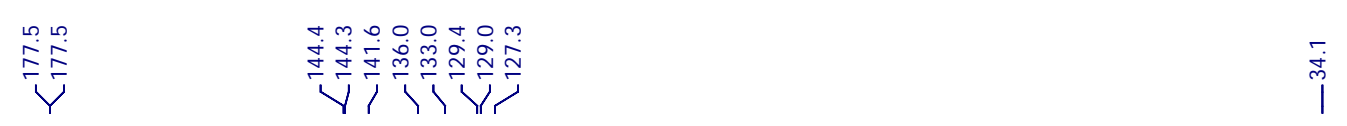

12

${ }^{13} \mathrm{C}\left\{{ }^{1} \mathrm{H}\right\}$ NMR

$\left(125 \mathrm{MHz}, \mathrm{CDCl}_{3}, 23^{\circ} \mathrm{C}\right)$

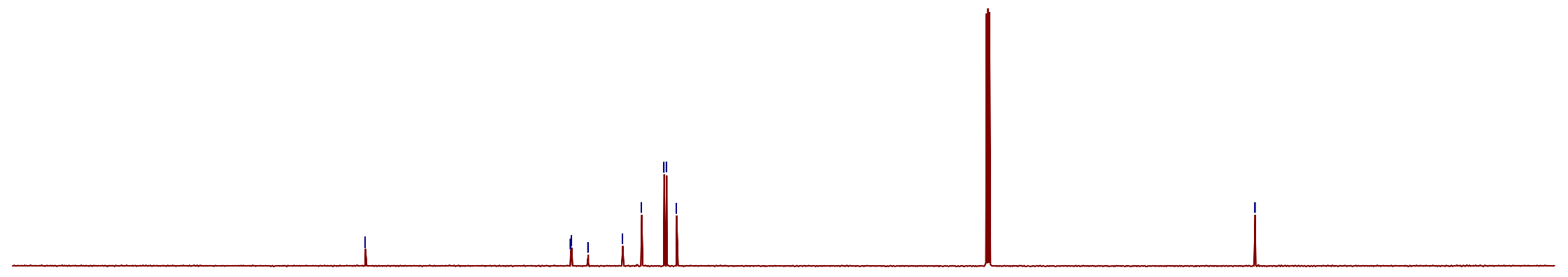

$\begin{array}{llllllllllllllllllllllllllllllll}230 & 220 & 210 & 200 & 190 & 180 & 170 & 160 & 150 & 140 & 130 & 120 & 110 & 100 & 90 & 80 & 70 & 60 & 50 & 40 & 30 & 20 & 10 & 0 & -10\end{array}$ 


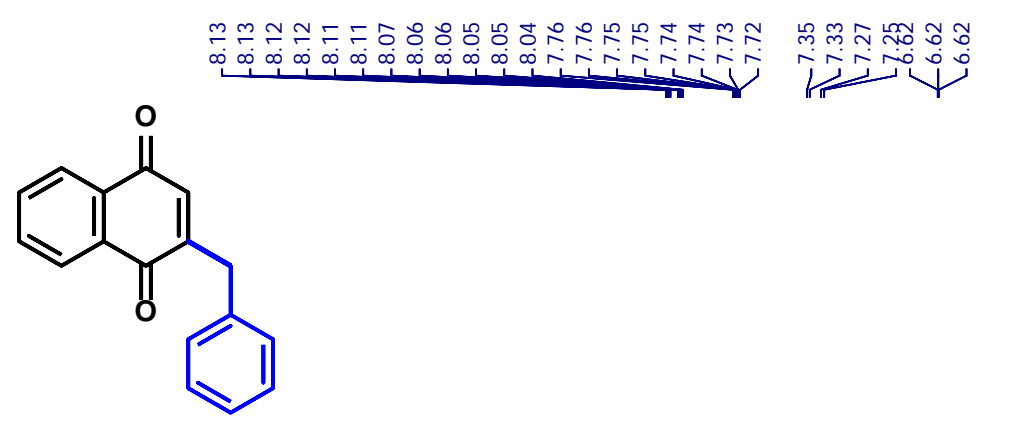

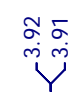

13

${ }^{1}$ H NMR

(500 MHz, $\mathrm{CDCl}_{3}, 23^{\circ} \mathrm{C}$ )

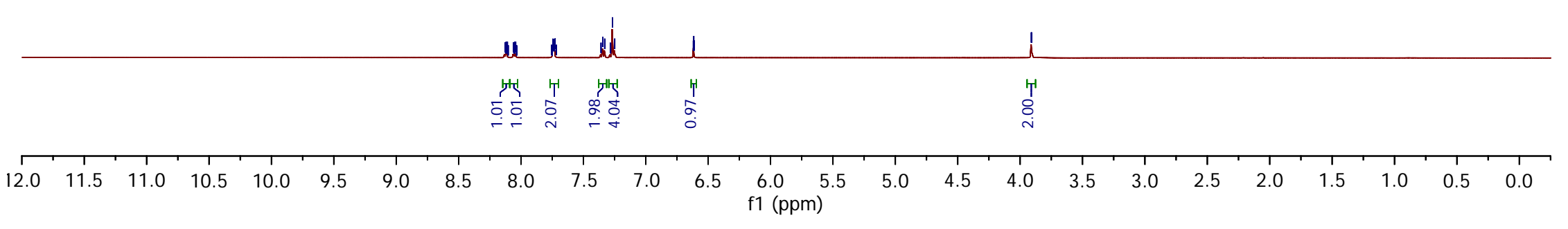




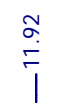

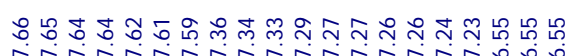

ก

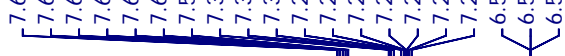<smiles>O=C1C(Cc2ccccc2)=CC(=O)c2c(O)cccc21</smiles>

14-C2

${ }^{1} \mathrm{H}$ NMR

(500 MHz, $\mathrm{CDCl}_{3}, 23^{\circ} \mathrm{C}$ )

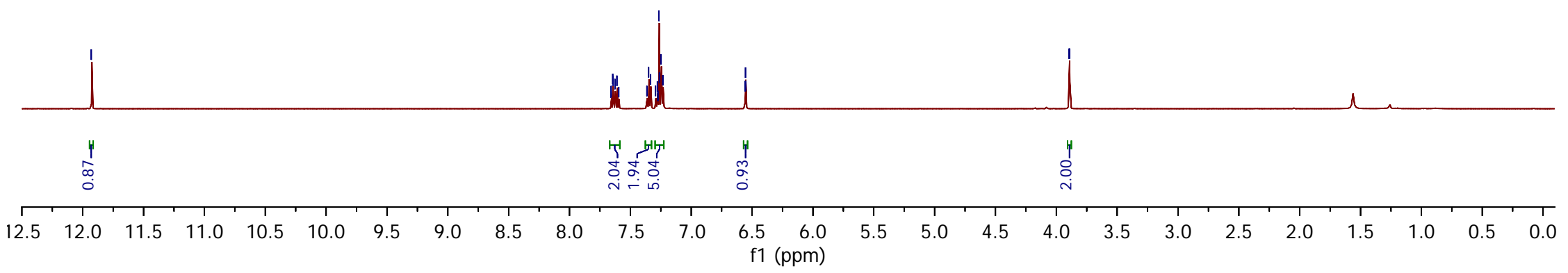


S44

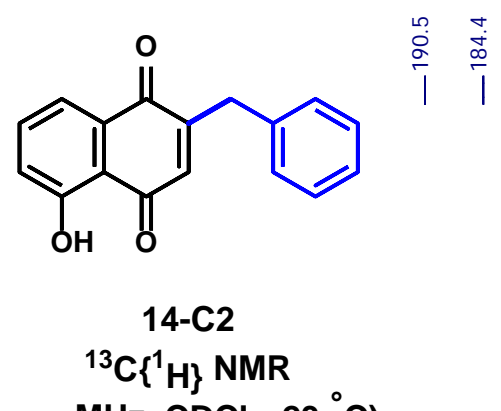

(125 $\mathrm{MHz}, \mathrm{CDCl}_{3}, 23^{\circ} \mathrm{C}$ ) l

$\stackrel{\substack{p \\ m}}{m}$
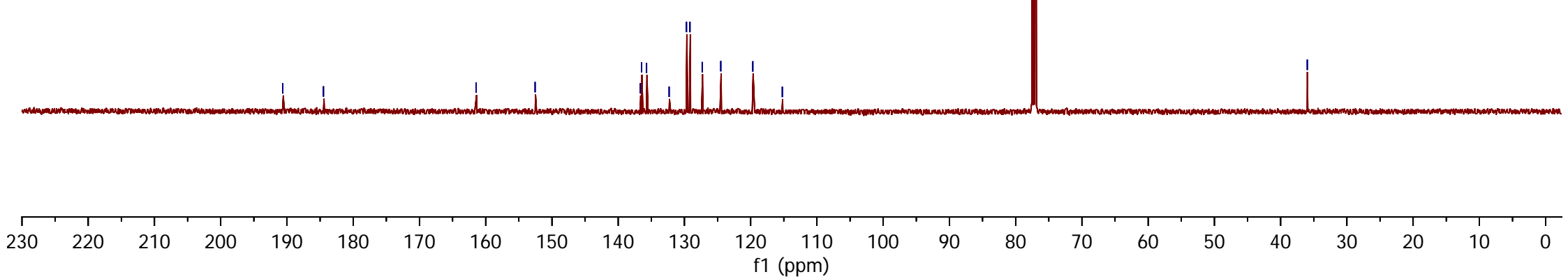


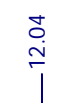

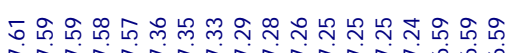

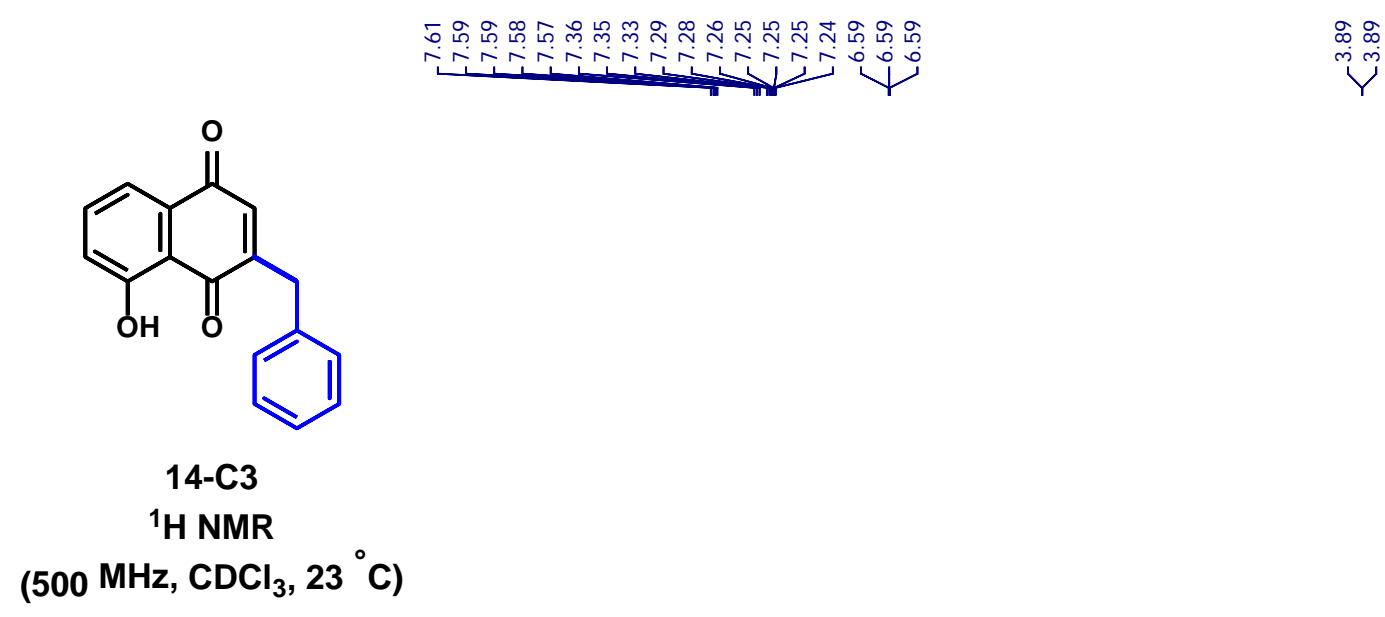

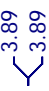

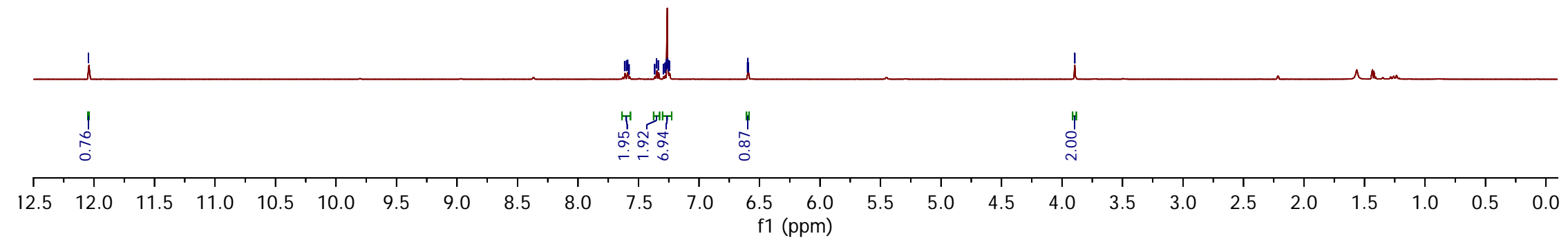




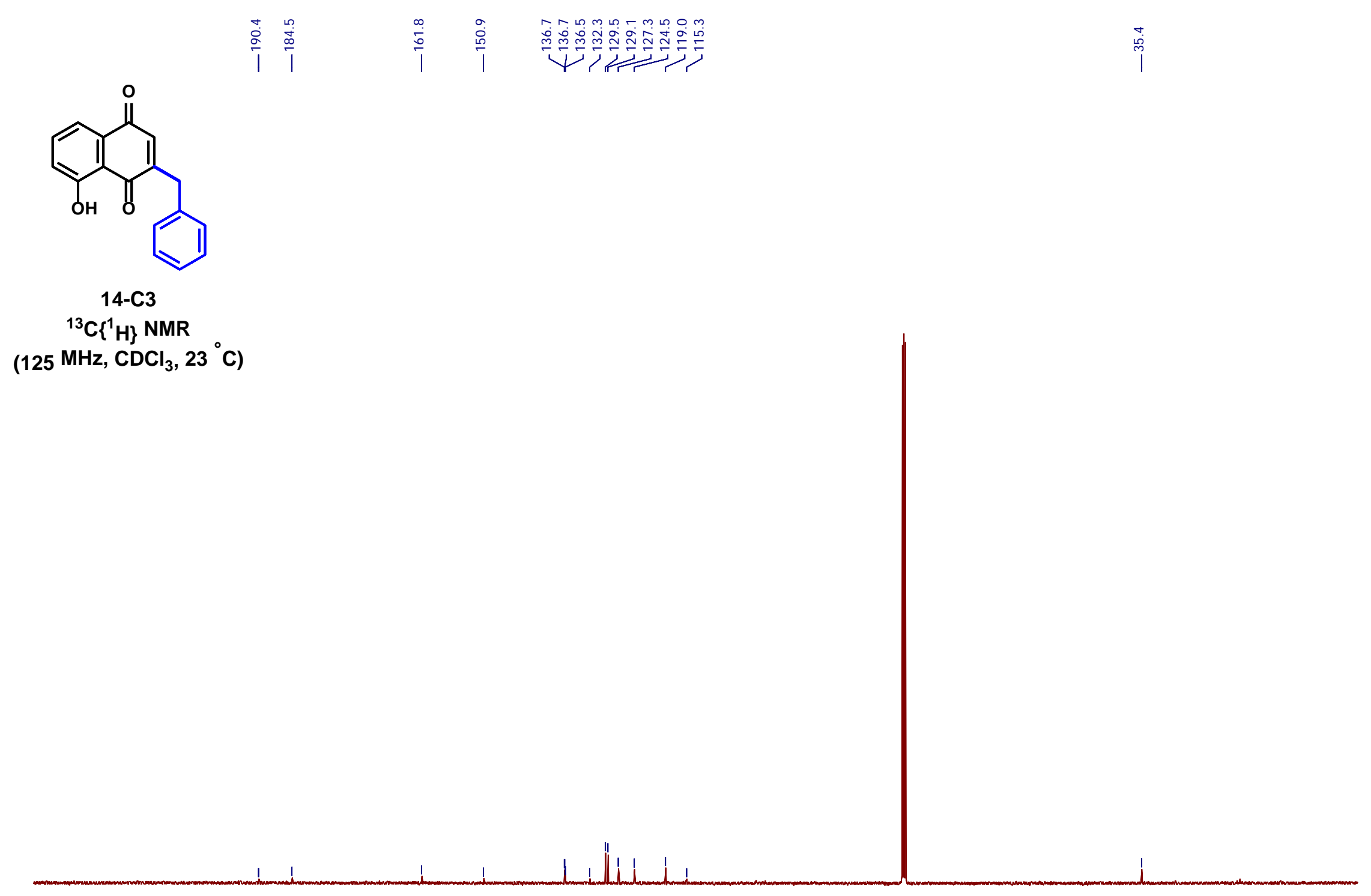

$\begin{array}{rrrrrrrrrrrrrrrrrrrrrrrrrrrrrrr} & 230 & 220 & 210 & 200 & 190 & 180 & 170 & 160 & 150 & 140 & 130 & \begin{array}{r}120 \\ \mathrm{f} 1(\mathrm{ppm})\end{array} & 110 & 90 & 80 & 70 & 60 & 50 & 40 & 30 & 20 & 10 & 0\end{array}$




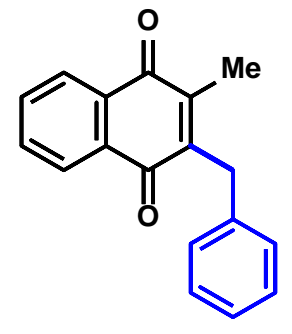

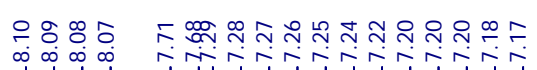

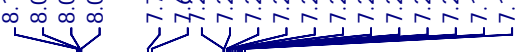

15

${ }^{1} \mathrm{H}$ NMR

(400 MHz, CDCl3, $23^{\circ} \mathrm{C}$ )

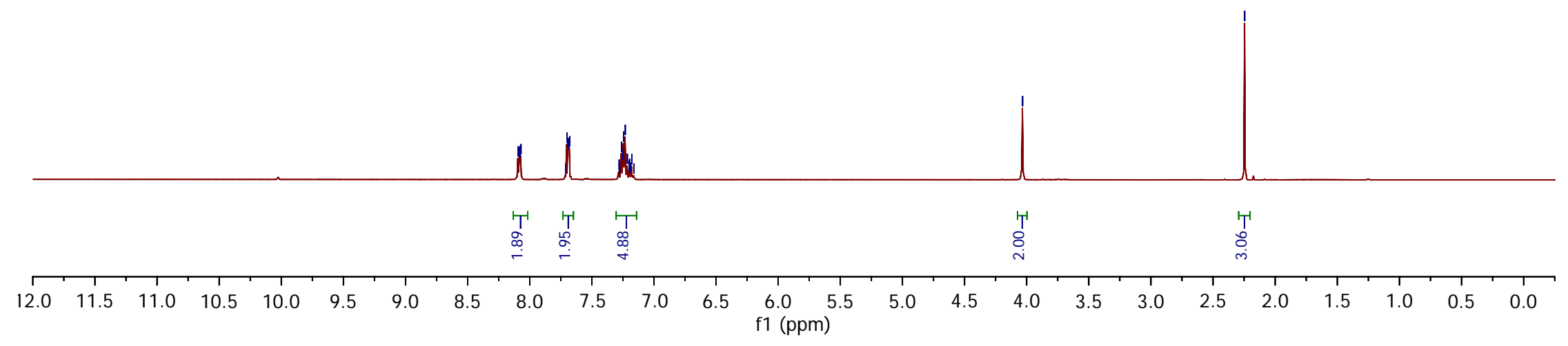




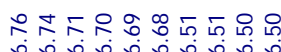

0.00000000

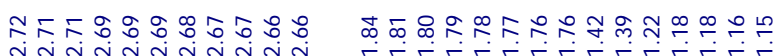

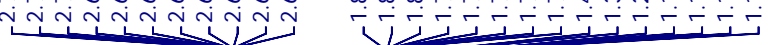

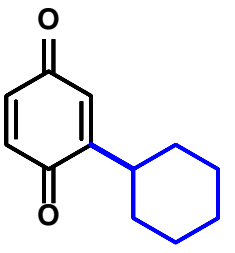

16

${ }^{1}{ }_{H}$ NMR

(500 MHz, $\mathrm{CDCl}_{3}, 23^{\circ} \mathrm{C}$ )

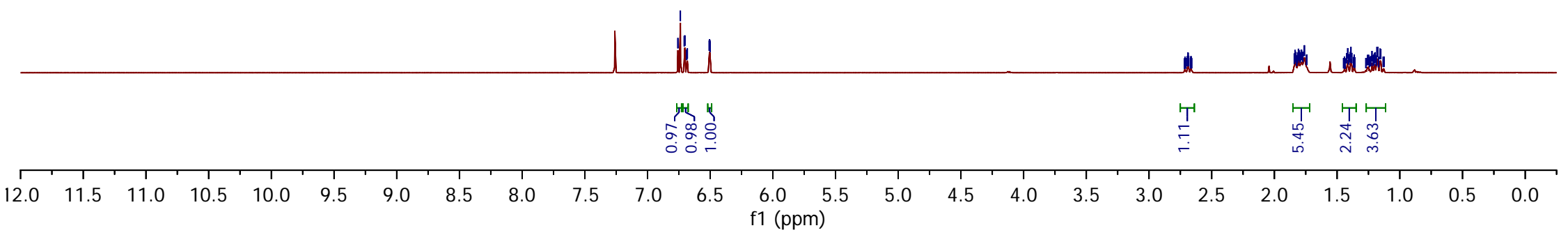




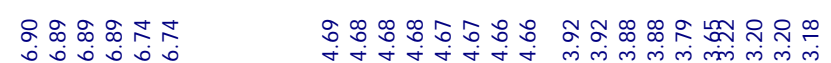

Y

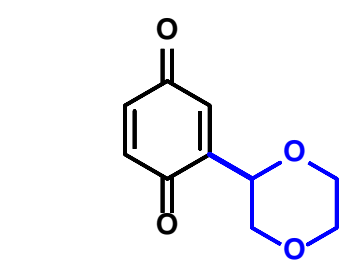

17

${ }^{1} \mathrm{H}$ NMR

(500 $\mathrm{MHz}, \mathrm{CDCl}_{3}, 23^{\circ} \mathrm{C}$ )

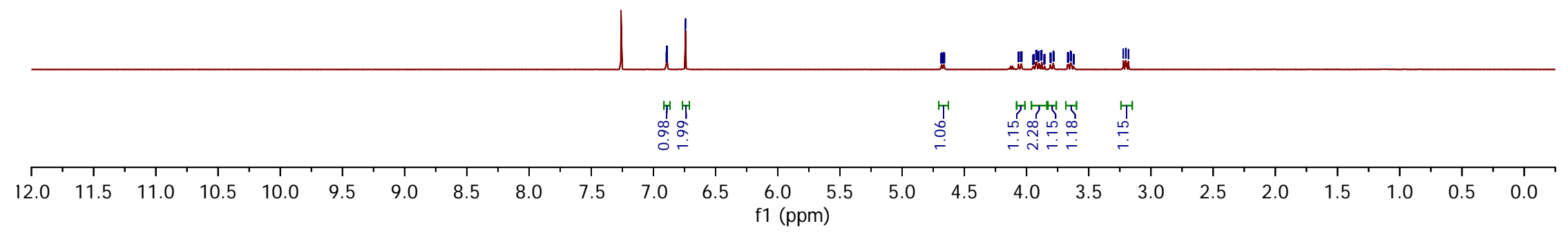




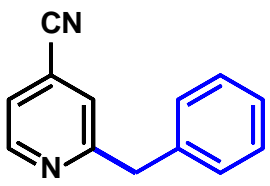

18

${ }^{1}$ H NMR

(500 $\mathrm{MHz}, \mathrm{CDCl}_{3}, 23^{\circ} \mathrm{C}$ )

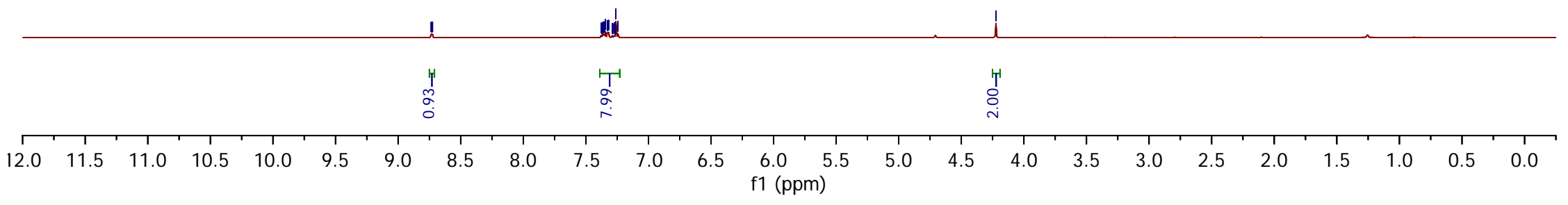




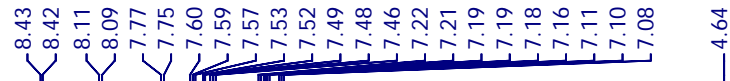

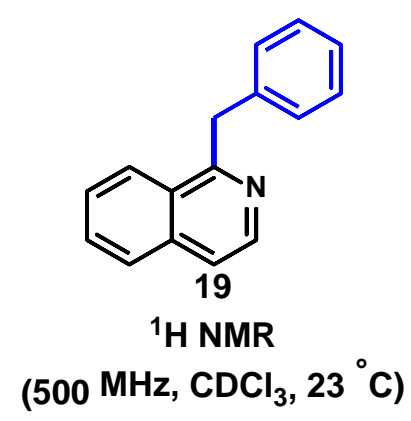

1. I L

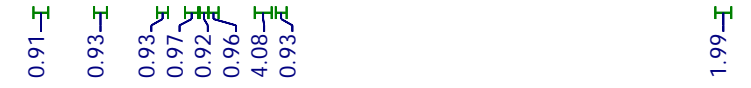

$\begin{array}{rllllllllllllllllllllllllll}12.0 & 11.5 & 11.0 & 10.5 & 10.0 & 9.5 & 9.0 & 8.5 & 8.0 & 7.5 & 7.0 & 6.5 & \begin{array}{c}6.0 \\ \mathrm{f} 1(\mathrm{ppm})\end{array} & 5.5 & 5.0 & 4.5 & 4.0 & 3.5 & 3.0 & 2.5 & 2.0 & 1.5 & 1.0 & 0.5 & 0.0\end{array}$




\section{References}

1. Galloway, J. D.; Mai, D. N.; Baxter, R. D. Silver-Catalyzed Minisci Reactions Using Selectfluor as a Mild Oxidant. Org. Lett. 2017, 19, 5772-5775.

2. Li, X.; Wang, H.-Y.; Shi, Z.-J. Transition-metal-free cross-dehydrogenative alkylation of pyridines under neutral conditions. New J. Chem. 2013, 37, 1704-1706.

3. Chu, H.; Sun, S.; Yu, J.-T.; Cheng, J. Rh-catalyzed sequential oxidative C-H activation/annulation with geminal-substituted vinyl acetates to access isoquinolines . Chem. Commun. 2015, 51, 13327. 